\title{
Semantics for a Quantum Programming Language by Operator Algebras
}

\author{
Kenta Cho \\ Institute for Computing and Information Sciences (iCIS) \\ Radboud University Nijmegen, The Netherlands \\ K.Cho@cs.ru.nl, http://www.cs.ru.nl/K.Cho/
}

\begin{abstract}
This paper presents a novel semantics for a quantum programming language by operator algebras, which are known to give a formulation for quantum theory that is alternative to the one by Hilbert spaces. We show that the opposite category of the category of $W^{*}$-algebras and normal completely positive subunital maps is an elementary quantum flow chart category in the sense of Selinger. As a consequence, it gives a denotational semantics for Selinger's first-order functional quantum programming language QPL. The use of operator algebras allows us to accommodate infinite structures and to handle classical and quantum computations in a unified way.
\end{abstract}

\section{Introduction}

Aiming at high-level and structured description of quantum computation/information, many quantum programming languages have been proposed and their semantics studied [12,44]. As one of pioneering works, in 2004 Selinger [39] proposed a first-order functional quantum programming language QPL (or QFC), and gave its denotational semantics rigorously in terms of categories. He (jointly with Valiron) successively started to study a higher-order quantum programming language, or the quantum lambda calculus [40-42]. It turned out to be challenging to give a denotational semantics for the quantum lambda calculus (with the full features, such as the ! modality and recursion). The first denotational semantics was given via Geometry of Interaction [16]; but there are several different approaches [24,33]. As is stated in [33, §1], the problem lies in that quantum computation is typically modelled by using finite dimensional Hilbert spaces, and hence it is difficult to model infinite structures in computation.

The present paper proposes a novel denotational semantics for a quantum programming language by operator algebras. Operator algebras, specifically $C^{*}$-algebras and $W^{*}$-algebras (the latter are also known as von Neumann algebras), give an alternative formulation for quantum theory (sometimes called the algebraic formulation [22]). It is worth mentioning that von Neumann himself, who formulated quantum theory by Hilbert spaces [31], developed the theory of operator algebras [26-30] (some of them jointly with Murray), and later preferred the algebraic approach for quantum theory [34]. Operator algebras have been successfully used in areas such as quantum statistical mechanics [3] and quantum field theory [ [2 13 [4]. They have also been of growing importance in the area of quantum information [20]; for example, in [8], the impossibility of quantum bit commitment is (re)examined in the algebraic formalism.

\section{Contributions and related work}

In this paper it is shown that the category $\mathbf{W s t a r}$ CPSU of $W^{*}$-algebras and normal completely positive subunital maps is a Dcppo ${ }_{\perp}$-enriched symmetric monoidal category with $\mathbf{D c p p o}_{\perp}$-enriched finite products. It follows that its opposite $\left(\mathbf{W s t a r}_{\mathrm{CPSU}}\right)^{\mathrm{op}}$ is an $\omega \mathbf{C p p o - e n r i c h e d ~ e l e m e n t a r y ~ q u a n t u m ~ f l o w ~ c h a r t ~}$ 
category. As a consequence, it gives rise to a denotational semantics for a first-order functional quantum programming language QPL designed by Selinger [39].

Selinger himself gave a denotational semantics for QPL by the category $\mathbf{Q}$ [39]. In comparison to his original semantics, our semantics by operator algebras has the following two advantages. First, our semantics accommodates infinite structures such as a type nat of natural numbers. This is because we discuss general $W^{*}$-algebras and do not restrict them to finite dimensional ones. In $\$ 8$ we will see that our model can be considered as an infinite dimensional extension of Selinger's model. Second, it is known that commutative $C^{*}$-algebras (resp. $W^{*}$-algebras) correspond to and certain type of topological (resp. measure) spaces, and therefore we can interpret classical computation as a map between commutative algebras. This correspondence (or duality) is studied well for $C^{*}$-algebras e.g. in [11], but not yet very well for $W^{*}$-algebras. In this paper, hence, we restrict it to deterministic computation (in the category Set), and give rudimentary results in $\$ 9$ as a first step. It will enable us to handle classical and quantum computations in a unified way. Although the present paper has not yet been able to give a semantics for a higher-order quantum programming language, compared to such works [16, 24, 33], our semantics is quite simple: a type is interpreted just as a $W^{*}$-algebra, and a program as a map of them (in opposite direction). However, the theory of operator algebras itself could be complicated enough.

As is the case in previous works [16, 24, 33, 39], quantum computation is usually modelled by using finite dimensional Hilbert spaces $\mathbb{C}^{n}$ (or matrix algebras $\mathcal{M}_{n} \cong \mathcal{B}\left(\mathbb{C}^{n}\right)$ ). It seems that the (explicit) use of operator algebras is not so common in the area of quantum computation. Recently there are several works using $C^{*}$-algebras [11 19], which led the author to the present work. The use of $W^{*}$-algebras in this context appeared independently and coincidentally in Rennela's thesis [35] and the present work (or the author's thesis [6]). In [35, Theorem 3.8], he also observed that the category Wstar PSU of $W^{*}$-algebras and normal positive subunital maps are Dcppo-enriched, which is almost the same result as Theorem 5.3 in the present paper. In his latest paper [36], he further showed that Wstar PSU is algebraically compact for a certain class of functors. This result enables us to have inductively defined types.

Some related technical results have appeared in [5], which studies spaces of maps ('quantum operations') between $W^{*}$-algebras, and maps ('quantum supermaps') between them. For instance, [5, Proposition 7] states (in our terms) that $\mathbf{W s t a r}_{\mathrm{CP}}(M, N)$ is bounded directed complete.

\section{Organisation of the paper}

First we review complete partial orders in $\$ 2$, and then review Selinger's work on QPL in $\$ 3$. In $\$ 4$ we also review the basics of operator algebras, and then look at the order-theoretic perspective of operator algebras. We show in $\$ 5$ that $\mathbf{W s t a r}_{\mathrm{CPSU}}$ is a Dcppo ${ }_{\perp}$-enriched symmetric monoidal category with Dcppo $_{\perp}$-enriched finite products and in $\$ 6$ that its opposite $\left(\mathbf{W s t a r}_{\mathrm{CPSU}}\right)^{\mathrm{op}}$ is an $\omega \mathbf{C p p o - e n r i c h e d ~ e l e - ~}$ mentary quantum flow chart category, hence gives a denotational semantics for QPL. Section 7 discuss a duality between Selinger's and our semantics, and in $\$ 8$ it is shown that Selinger's model is contravariantly embedded into our model. In $\$ 9$ we see that our model can accommodate infinite types, and also classical computation as commutative structures. Section 10 concludes the paper with future work.

This paper is based on the author's master thesis [6], in which one may find further details.

\section{Complete partial orders}

In this section, we will briefly review the notion of complete partial orders, which is essential in domain theory [1], and is fundamental for the denotational semantics of programming languages. 
Definition 2.1. A poset is

1. directed complete if every directed subset has a supremum;

2. bounded directed complete if every directed subset that is bounded from above has a supremum;

3. $\omega$-complete if every $\omega$-chain $\left(\left(x_{n}\right)_{n \in \omega}\right.$ with $\left.x_{n} \leq x_{n+1}\right)$ has a supremum;

4. pointed if it has a least element (denoted by $\perp$ ).

A (bounded) directed complete poset is abbreviated as a (b)dcpo, and an $\omega$-complete poset as a $\omega c p o$.

Definition 2.2. A map between posets (in 3, pointed posets) is

1. Scott-continuous if it preserves suprema of directed subsets;

2. $\omega$-(Scott-)continuous if it preserves suprema of $\omega$-chains;

3. strict if it preserves the least element.

Note that every dcpo is an $\omega$ cpo, and every Scott-continuous map is $\omega$-continuous. The next theorem is a fundamental tool to give an interpretation of a program with recursion and loop.

Theorem 2.3. Every $\omega$-continuous endomap $f$ on a pointed $\omega$ cpo has a least fixed point, which is given by $\bigvee_{n} f^{n}(\perp)$.

We here fix the notations of categories we use in this paper.

Definition 2.4. We denote by Dcppo $_{\perp}$ the category of pointed dcpos and strict Scott-continuous maps, and by $\omega \mathbf{C p p o}$ the category of pointed $\omega$ cpos and $\omega$-continuous maps.

Both categories Dcppo $\perp$ and $\omega \mathbf{C p p o}$ have products, which are given by cartesian products of underlying sets with coordinatewise order. When we speak of Dcppo $\perp^{-}$( or $\omega \mathbf{C p p o}$ )-enrichment of categories, these cartesian structures are taken as monoidal structures.

\section{Selinger's QPL and its semantics}

In [39], Selinger proposed a quantum programming language QPL and its denotational semantics. It is a first-order functional language with loop and recursion, and is described by flow chart syntax, besides by textual syntax ${ }^{1}$ He showed a denotational semantics for QPL is given by the following category.

Definition 3.1 ([39, §6.6]). An elementary quantum flow chart category is a symmetric monoidal category $(\mathbf{C}, \otimes, I)$ with traced finite coproducts $(\oplus, 0, \operatorname{Tr})$ such that:

- For each $A \in \mathbf{C}, A \otimes(-)$ is a traced monoidal functor.

- $\mathbf{C}$ has a distinguished object qbit with arrows $\imath: I \oplus I \rightarrow$ qbit and $p:$ qbit $\rightarrow I \oplus I$ such that $p \circ \imath=\mathrm{id}$.

Theorem 3.2 ([39, §6.6]). Let $\mathbf{C}$ be an elementary quantum flow chart category. Suppose we have an assignment $\eta$ of built-in unitary operator symbols $S$ of arity $n$ to arrows $\eta_{S}: \mathbf{q b i t}^{\otimes n} \rightarrow \mathbf{q b i t}^{\otimes n}$ in $\mathbf{C}$. Then we have an interpretation of $Q P L$ programs without recursion. If $\mathbf{C}$ is additionally $\omega \mathbf{C p p o - e n r i c h e d , ~ t h e n ~}$ we can also interpret $Q P L$ programs with recursion.

Selinger also gave a concrete model for QPL, constructing an $\omega \mathbf{C p p o - e n r i c h e d ~ e l e m e n t a r y ~ q u a n t u m ~ f l o w ~}$ chart category $\mathbf{Q}$ as follows.

\footnotetext{
${ }^{1}$ Strictly speaking, the name QPL is reserved for the textual syntax. In the present paper we do not distinguish the two syntaxes because semantics are given in the same manner.
} 
Definition 3.3. We write $\mathcal{M}_{n}$ for the set of complex $n \times n$ matrices.

1. The category $\mathbf{C P M}_{s}$ is defined as follows.

- An object is a natural number.

- An arrow $f: n \rightarrow m$ is a completely positive map $f: \mathcal{M}_{n} \rightarrow \mathcal{M}_{m}$.

2. The category $\mathbf{C P M}$ is the finite biproduct completion of $\mathbf{C P M}$. Specifically:

- An object is a sequence $\vec{n}=\left(n_{1}, \ldots, n_{k}\right)$ of natural numbers.

- An arrow $f: \vec{n} \rightarrow \vec{m}$ is a matrix $\left(f_{i j}\right)$ of arrows $f_{i j}: n_{j} \rightarrow m_{i}$ in $\mathbf{C P M}_{s}$.

3. The category $\mathbf{Q}$ is a subcategory of $\mathbf{C P M}$ such that

- Objects are the same as CPM.

- An arrow is $f: \vec{n} \rightarrow \vec{m}$ in $\mathbf{C P M}$ which is trace-nonincreasing, i.e.

$$
\sum_{i} \sum_{j} \operatorname{tr}\left(f_{i j}\left(A_{j}\right)\right) \leq \sum_{j} \operatorname{tr}\left(A_{j}\right)
$$

for all $\left(A_{j}\right)_{j}$ with positive $A_{j} \in \mathcal{M}_{n_{j}}$.

The category $\mathbf{Q}$ turns out to have the monoidal structure $(\otimes, I)$ and finite coproducts $(\oplus, 0)$, and furthermore to be $\omega \mathbf{C p p o - e n r i c h e d . ~ T h e ~ c a t e g o r y ~ i s ~ a l s o ~ e q u i p p e d ~ w i t h ~ a ~ m o n o i d a l ~ t r a c e ~ w . r . t . ~}(\oplus, 0)$, which is obtained from its $\omega \mathbf{C p p o - e n r i c h e d ~ s t r u c t u r e . ~ T h e n ~ i t ~ f o r m s ~ a n ~} \omega \mathbf{C p p o - e n r i c h e d ~ e l e m e n t a r y ~ q u a n t u m ~}$ flow chart category with qbit $:=2$. As Selinger mentioned [39, §6.4] (though he did not give a proof), the construction of monoidal trace from $\omega \mathbf{C p p o - e n r i c h e d ~ s t r u c t u r e ~ w o r k s ~ f o r ~ e v e r y ~} \omega \mathbf{C p p o - e n r i c h e d ~ c a t e - ~}$ gory with finite coproducts (satisfying certain condition). Specifically, we have the following theorem.

\section{Theorem 3.4.}

1. Every $\omega \mathbf{C p p o - e n r i c h e d ~ c o c a r t e s i a n ~ c a t e g o r y ~ w i t h ~ r i g h t - s t r i c t ~ c o m p o s i t i o n ~ ( i . e . ~} f \circ \perp=\perp$ ) is traced.

2. Let $\mathbf{C}$ and $\mathbf{D}$ be $\omega \mathbf{C p p o - e n r i c h e d ~ c o c a r t e s i a n ~ c a t e g o r i e s ~ w i t h ~ r i g h t - s t r i c t ~ c o m p o s i t i o n , ~ w h i c h ~ a r e ~}$ traced by 1. Every $\omega \mathbf{C p p o - e n r i c h e d ~ c o c a r t e s i a n ~ f u n c t o r ~ b e t w e e n ~} \mathbf{C}$ and $\mathbf{D}$ satisfying $F \perp=\perp$ is traced.

Here, a cocartesian category refers to a monoidal category whose monoidal structure is given by finite coproducts. The proof is found in Appendix A. Combining Theorem 3.4 with Definition 3.1, we obtain a sufficient condition for a category to be an $\omega \mathbf{C p p o - e n r i c h e d ~ e l e m e n t a r y ~ q u a n t u m ~ f l o w ~ c h a r t ~ c a t e g o r y , ~}$ which gives a semantics for QPL programs with recursion.

Theorem 3.5. A category is an $\omega \mathbf{C p p o - e n r i c h e d ~ e l e m e n t a r y ~ q u a n t u m ~ f l o w ~ c h a r t ~ c a t e g o r y ~ i f ~ i t ~ i s ~ a n ~}$ $\omega \mathbf{C p p o - e n r i c h e d ~ s y m m e t r i c ~ m o n o i d a l ~ c a t e g o r y ~}(\mathbf{C}, \otimes, I)$ satisfying the following conditions.

- C has $\omega \mathbf{C p p o - e n r i c h e d ~ f i n i t e ~ c o p r o d u c t s ~}(\oplus, 0)$;

- the composition is right-strict;

- for each $A \in \mathbf{C}$, a functor $A \otimes(-)$ preserves finite coproducts and bottom arrows;

- C has a distinguished object qbit with arrows $\imath: I \oplus I \rightarrow$ qbit and $p:$ qbit $\rightarrow I \oplus I$ such that $p \circ \imath=\mathrm{id}$. 


\section{Operator algebras: $C^{*}$-algebras and $W^{*}$-algebras}

\section{1 $\quad C^{*}$-algebras and $W^{*}$-algebras}

The theory of operator algebras is usually concerned with $C^{*}$-algebras and $W^{*}$-algebras (the latter are often studied as von Neumann algebras). Due to limitations of space, here we just fix notations and terminology, and list the basic results. A reader who is not familiar with these topics may consult [38,43] for the standard theory and [21,25] for the categorical perspective.

In this paper, $C^{*}$-algebras are always assumed to be unital. Note also that a 'map' usually refers to a linear map. For a $C^{*}$-algebra $A$, we write $A_{\mathrm{sa}}$ for the set of self-adjoint elements, and $[0,1]_{A}:=\{x \in A \mid$ $0 \leq x \leq 1\}$ for the "unit interval", or the set of effects. We write Cstar for the category of $C^{*}$-algebras and linear maps. We will denote subcategories of Cstar, with the same objects but different maps, by adding subscripts to Cstar as follows: $\mathrm{M}$ for 'multiplicative'; I for 'involutive'; $\mathrm{P}$ for 'positive'; CP for 'completely positive'; U for 'unital'; SU for 'subunital'2. For example, Cstar MIU $_{\text {is }}$ the category of $C^{*}$-algebras and unital $*$-homomorphisms (i.e. multiplicative involutive maps), while Cstar $_{\mathrm{CPSU}}$ is the category of $C^{*}$-algebras and completely positive subunital maps. Note that there are inclusions like Cstar $_{\mathrm{MI}} \subseteq$ Cstar $_{\mathrm{CP}} \subseteq$ Cstar $_{\mathrm{P}}$

A $W^{*}$-algebra is a $C^{*}$-algebra that has a (necessarily unique) predual. Every $W^{*}$-algebra is equipped with the weak* topology introduced by its predual, which is called the ultraweak topology. A map between $W^{*}$-algebras is said to be normal if it is ultraweakly continuous. We write Wstar for the category of $W^{*}$-algebras and normal maps. We denote subcategories of Wstar in the same manner as Cstar. For example, Wstar MIU $_{\text {is }}$ the category of $W^{*}$-algebras and normal unital $*$-homomorphisms, which is a non-full subcategory of $\mathbf{C s t a r}_{\mathrm{MIU}}$ (since maps in Wstar are required to be normal).

We denote direct sum [38, Definition 1.1.5] of $C^{*}$ - and $W^{*}$-algebras by $\oplus$. It forms categorical

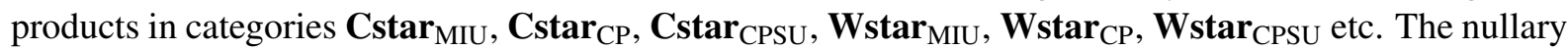
direct sum is the zero space 0 .

There are several kinds of tensor products for $C^{*}$ - and $W^{*}$-algebras. In this paper we use the spatial $C^{*}$-tensor product [38, Definition 1.22.8], denoted by $\otimes$, for $C^{*}$-algebras, and the spatial $W^{*}$-tensor product [38, Definition 1.22.10], denoted by $\bar{\otimes}$, for $W^{*}$-algebras. The spatial $C^{*}$-tensor product makes

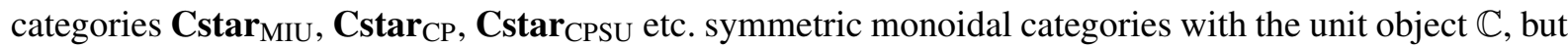

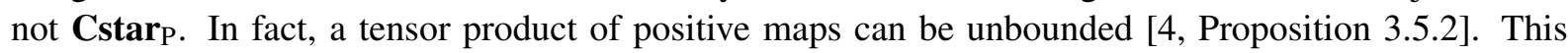
is why we need the notion of the complete positivity. Similarly, the spatial $W^{*}$-tensor product makes

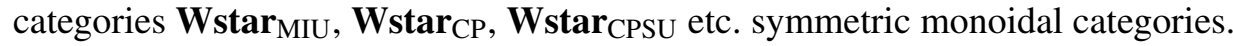

The spatial $C^{*}$ - and $W^{*}$-tensor products distribute over finite direct sums, i.e.

$$
A \otimes(B \oplus C) \cong(A \otimes B) \oplus(A \otimes C), \quad A \otimes 0 \cong 0, \quad M \bar{\otimes}(N \oplus L) \cong(M \bar{\otimes} N) \oplus(M \bar{\otimes} L), \quad M \bar{\otimes} 0 \cong 0,
$$

for $C^{*}$-algebras $A, B, C$ and $W^{*}$-algebras $M, N, L$. These properties seem to be known results, but to be missing in the standard literature. For the sake of completeness, the proofs are included in Appendix B For our purpose, it is useful to restate them as follows.

\section{Proposition 4.1.}

1. For each $C^{*}$-algebra $A$, a functor $A \otimes(-)$ on $\mathbf{C s t a r}_{\mathrm{MIU}}$ preserves finite products.

2. For each $W^{*}$-algebra $M$, a functor $M \bar{\otimes}(-)$ on $\mathbf{W s t a r}_{\mathrm{MIU}}$ preserves finite products.

\footnotetext{
${ }^{2}$ A map $f$ between $C^{*}$-algebras is said to be subunital if $f(1) \leq 1$. Note that in the author's thesis $[6]$ (and the preliminary version of this paper), 'pre-unital' is used instead of 'subunital'.
} 


\subsection{Order theory in operator algebras}

Recall each $C^{*}$-algebra is equipped with a partial order $\leq$ defined by: $a \leq b \Longleftrightarrow$ ' $b-a$ is positive'. Many concepts on operator algebras can be rephrased in terms of the orders. Observe, for instance, the following easy proposition.

Proposition 4.2. A map between $C^{*}$-algebras is positive if and only if it is monotone.

In fact, the orders on $W^{*}$-algebras have a very nice property, called monotone completeness, which distinguishes $W^{*}$-algebras from $C^{*}$-algebras.

Definition 4.3. A $C^{*}$-algebra $A$ is monotone complete (or monotone closed) if every norm-bounded directed subset of $A_{\mathrm{sa}}$ has the supremum in $A_{\mathrm{sa}}$.

Proposition 4.4 ([38, Lemma 1.7.4]). Every $W^{*}$-algebra is monotone complete. Moreover, suprema are obtained as ultraweak limits.

We can also rephrase the notion of normality of positive (i.e. monotone) maps between $W^{*}$-algebras.

Proposition 4.5 ([7, Corollary 46.5]). Let $f: M \rightarrow N$ be a positive map between $W^{*}$-algebra. Then $f$ is normal (i.e. ultraweakly continuous) if and only if it preserves the supremum of every norm-bounded directed subset of $M_{\mathrm{sa}}$.

Therefore, we shall say a positive map $f: A \rightarrow B$ between monotone complete $C^{*}$-algebras is normal if it preserves the supremum of every norm-bounded directed subset of $A_{\text {sa }}$. Then $W^{*}$-algebras can be characterised as follows.

Theorem 4.6 ([43, Theorem III.3.16]). A $C^{*}$-algebra is a $W^{*}$-algebra if and only if it is monotone complete and admits sufficiently many normal positive functionals (i.e. they separate the points).

Now, we shall recapture the order structures in operator algebras from a more order-theoretic (or domain-theoretic [1]) point of view.

Proposition 4.7. Let $A$ be a $C^{*}$-algebra. The following are equivalent.

1. A is monotone complete.

2. $A_{\mathrm{sa}}$ is bounded directed complete.

3. $[0,1]_{A}$ is directed complete.

Proof. Without loss of generality, we may assume directed subsets are bounded from below. Then, $1 \Longleftrightarrow$ 2 follows from the fact that norm-boundedness and order-boundedness coincide (see Proposition C.4 in Appendix C).

$2 \Longrightarrow 3$ is trivial. For the converse, note that $A_{\mathrm{sa}}$ is an ordered vector space over $\mathbb{R}$. Hence we can obtain the supremum of a bounded directed subset of $A_{\mathrm{sa}}$ by transforming it into a directed subset of $[0,1]_{A}$ by shifting and scaling.

Consequently, for every $W^{*}$-algebras $M, M_{\text {sa }}$ is a bdcpo, and $[0,1]_{M}$ is a pointed dcpo. We have a corresponding result for normal maps, which is proved in a similar way, using Proposition 4.5.

Proposition 4.8. Let $f: M \rightarrow N$ be a positive map between $W^{*}$-algebra. The first two of the following are equivalent. They are also equivalent to the third when $f$ is subunital.

1. $f$ is normal.

2. The restriction $\left.f\right|_{M_{\mathrm{sa}}}: M_{\mathrm{sa}} \rightarrow N_{\mathrm{sa}}$ is Scott-continuous.

3. The restriction $\left.f\right|_{[0,1]_{M}}:[0,1]_{M} \rightarrow[0,1]_{N}$ is Scott-continuous. 


\section{Dcppo $_{\perp}$-enrichment of the category of $W^{*}$-algebras}

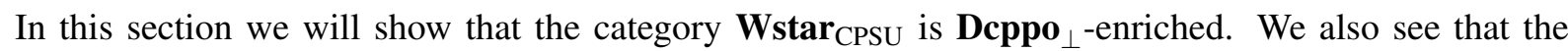
monoidal product $(\bar{\otimes}, \mathbb{C})$ and finite products $(\oplus, 0)$ on $\mathbf{W s t a r}_{\mathrm{CPSU}}$ are $\mathbf{D c p p o}_{\perp}$-enriched.

Definition 5.1. Let $M, N$ be $W^{*}$-algebras. We define a partial order $\sqsubseteq$ on $\operatorname{Wstar}_{\text {CPSU }}(M, N)$ by

$$
f \sqsubseteq g \stackrel{\text { def }}{\Longleftrightarrow} g-f \text { is completely positive . }
$$

Proposition 5.2. For any $W^{*}$-algebras $M$ and $N, \mathbf{W s t a r}_{\mathrm{CPSU}}(M, N)$ with the order $\sqsubseteq$ is a pointed dcpo.

Proof. First of all, it is easy to see that the zero map is the least element of $\operatorname{Wstar}_{\mathrm{CPSU}}(M, N)$, hence it is pointed.

Note that a positive subunital map $f: A \rightarrow B$ between $C^{*}$-algebras restricts to $[0,1]_{A} \rightarrow[0,1]_{B}$, while a "linear' ${ }^{3}$ map $g:[0,1]_{A} \rightarrow[0,1]_{B}$ extends to a positive subunital map $A \rightarrow B$. Hence such maps correspond bijectively (cf. [11, Lemma 2]).

Let $\left(f_{i}\right)$ be a monotone net in $\mathbf{W s t a r}_{\mathrm{CPSU}}(M, N)$. For each $x \in[0,1]_{M},\left(f_{i}(x)\right)$ is a monotone net in $[0,1]_{N}$, which is a dcpo. Hence define $f(x):=\sup f_{i}(x)$. It is easy to see $f$ is a "linear" map $[0,1]_{M} \rightarrow$ $[0,1]_{N}$, so that we obtain a positive subunital map $f: M \rightarrow N$.

Normality of $f$ is proved as follows. For a monotone net $\left(x_{j}\right)$ in $[0,1]_{M}$,

$$
f\left(\sup x_{j}\right)=\sup _{i} f_{i}\left(\sup _{j} x_{j}\right)=\sup _{i}\left(\sup _{j} f_{i}\left(x_{j}\right)\right)=\sup _{j}\left(\sup _{i} f_{i}\left(x_{j}\right)\right)=\sup _{j} f\left(x_{j}\right) .
$$

Note that we can exchange of the order of sup ([1, Proposition 2.1.12]). Therefore $f:[0,1]_{M} \rightarrow[0,1]_{N}$ is Scott-continuous, and $f: M \rightarrow N$ is normal by Proposition 4.8 .

To show that $f$ is completely positive (hence $f \in \mathbf{W s t a r}_{\mathrm{CPSU}}(M, N)$ ) and that $f$ is indeed the supremum of $\left(f_{i}\right)_{i}$, we need some more results on $W^{*}$-algebras. The remaining proof is found at Proposition C.11 in Appendix C




now we prove:

Theorem 5.3. The category Wstar $\mathbf{C P S U}_{\text {is }} \mathbf{D c p p o}_{\perp}$-enriched. Moreover, the composition is bi-strict, i.e. $\perp \circ f=\perp$ and $f \circ \perp=\perp$ for each arrow $f$.

Moreover, finite products $(\oplus, 0)$ and the monoidal product $(\otimes, 0)$ are also suitably enriched:

Theorem 5.4. Finite products in $\mathbf{W s t a r}_{\mathrm{CPSU}}$ are $\mathbf{D c p p o}_{\perp}$-enriched.

Theorem 5.5. The symmetric monoidal product $(\bar{\otimes}, \mathbb{C})$ on $\mathbf{W s t a r}_{\mathrm{CPSU}}$ is $\mathbf{D c p p o}_{\perp}$-enriched. Moreover, the tensor product of maps is bi-strict, i.e. $\perp \bar{\otimes} f=\perp$ and $f \bar{\otimes} \perp=\perp$.

The details are found in Propositions C.14 and C.15 in Appendix C, respectively.

Remark 5.6. It is worth noting that the category Cstar $_{\mathrm{CPSU}}$ is never Dcppo ${ }_{\perp}$-enriched, nor $\omega \mathbf{C p p o}$ enriched. This is because we have an order-isomorphism $\operatorname{Cstar}_{\mathrm{CPSU}}(\mathbb{C}, A) \cong[0,1]_{A}$, whereas there exists a $C^{*}$-algebra such that $[0,1]_{A}$ is not $\omega$-complete (take $A=C([0,1])$ for example).

$$
{ }^{3} g(0)=0, g(x+y)=g(x)+g(y) \text { for } x+y \leq 1, g(r x)=r g(x) \text { for } r \in[0,1] .
$$




\section{Semantics for QPL by $W^{*}$-algebras}

We have proved that $\mathbf{W s t a r}_{\mathrm{CPSU}}$ is an $\mathbf{D c p p o}_{\perp}$-enriched symmetric monoidal category with $\mathbf{D c p p o}_{\perp^{-}}$ enriched finite products. Now we can show the following theorem.

Theorem 6.1. The opposite category of $\mathbf{W s t a r}_{\mathrm{CPSU}}$ is an $\omega \mathbf{C p p o - e n r i c h e d ~ e l e m e n t a r y ~ q u a n t u m ~ f l o w ~}$ chart category.

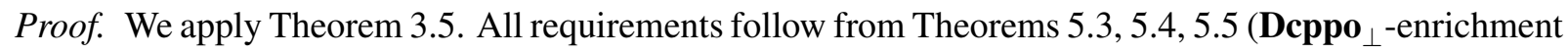
implies $\omega \mathbf{C p p o - e n r i c h m e n t ) , ~ a n d ~ P r o p o s i t i o n ~ 4 . 1 2 , ~ e x c e p t ~ a ~ d i s t i n g u i s h e d ~ o b j e c t ~ q b i t ~ w i t h ~ a r r o w s ~} \imath, p$.

We take qbit $:=\mathcal{M}_{2}$, the algebra of complex $2 \times 2$-matrices. We define two maps $\imath, p$ by

$$
\imath\left(\left[\begin{array}{cc}
x & y \\
z & w
\end{array}\right]\right)=(x, w), \quad p(x, y)=\left[\begin{array}{ll}
x & 0 \\
0 & y
\end{array}\right] .
$$

It is straightforward to see the two maps are positive, hence completely positive by [43, Corollary IV.3.5 and Proposition IV.3.9] (notice that $\mathbb{C} \oplus \mathbb{C}$ is commutative). They are normal because they are maps between finite dimensional $W^{*}$-algebras. Moreover they are clearly unital. Therefore $\imath$ and $p$ are arrows in Wstar ${ }_{\text {CPSU }}$. It is clear that $\imath \circ p=\mathrm{id}$, hence $p \circ \boldsymbol{l}=\mathrm{id}$ in $\left(\mathbf{W s t a r}_{\mathrm{CPSU}}\right)^{\mathrm{op}}$.

Every unitary operator $S:\left(\mathbb{C}^{2}\right)^{\otimes n} \rightarrow\left(\mathbb{C}^{2}\right)^{\otimes n}$ of arity $n$ determines an arrow $\eta_{S}:\left(\mathcal{M}_{2}\right)^{\bar{\otimes} n} \rightarrow\left(\mathcal{M}_{2}\right)^{\bar{\otimes} n}$ in Wstar CPSU $_{\text {by }}$

$$
\left(\mathcal{M}_{2}\right)^{\bar{\otimes} n} \cong \mathcal{M}_{2^{n}} \longrightarrow \mathcal{M}_{2^{n}} \cong\left(\mathcal{M}_{2}\right)^{\bar{\otimes} n}, \quad x \longmapsto S^{\dagger} x S
$$

where $S$ is seen as a complex $2^{n} \times 2^{n}$ matrix. By Theorem 3.2, finally, we show:

Theorem 6.2. The opposite category of $\mathbf{W s t a r}_{\mathrm{CPSU}}$ gives a denotational semantics for QPL (with recursion).

\section{Schrödinger vs. Heisenberg picture}

We have shown the category $\left(\mathbf{W s t a r}_{\mathrm{CPSU}}\right)^{\text {op }}$ gives a semantics for QPL. From now on we will discuss a comparison between two semantics by Selinger's $\mathbf{Q}$ and our $\left(\mathbf{W s t a r}_{\mathrm{CPSU}}\right)^{\text {op }}$.

Recall that for a Hilbert space $\mathcal{H}, \mathcal{B}(\mathcal{H})$, i.e. the set of bounded operators on $\mathcal{H}$, is a $W^{*}$-algebra with the predual $\mathcal{T}(\mathcal{H})$, i.e. the set of trace class operators on $\mathcal{H}$. For every normal map $\mathcal{E}: \mathcal{B}(\mathcal{H}) \rightarrow \mathcal{B}(\mathcal{K})$, there exists a corresponding bounded map $\mathcal{E}_{*}: \mathcal{T}(\mathcal{K}) \rightarrow \mathcal{T}(\mathcal{H})$ between preduals. They are related in the following way:

$$
\operatorname{tr}(\mathcal{E}(S) \cdot T)=\operatorname{tr}\left(S \cdot \mathcal{E}_{*}(T)\right)
$$

for all $S \in \mathcal{B}(\mathcal{H})$ and $T \in \mathcal{T}(\mathcal{K})$. Furthermore the following holds.

Proposition 7.1 ([17, §4.1.2]). Let $\mathcal{H}$ and $\mathcal{K}$ be Hilbert spaces. Suppose a normal map $\mathcal{E}: \mathcal{B}(\mathcal{H}) \rightarrow$ $\mathcal{B}(\mathcal{K})$ and a bounded map $\mathcal{E}_{*}: \mathcal{T}(\mathcal{K}) \rightarrow \mathcal{T}(\mathcal{H})$ related as above. Then

1. $\mathcal{E}$ is completely positive if and only if $\mathcal{E}_{*}$ is completely positive.

2. $\mathcal{E}$ is unital if and only if $\mathcal{E}_{*}$ is trace-preserving.

3. $\mathcal{E}$ is subunital if and only if $\mathcal{E}_{*}$ is trace-nonincreasing. 
Hence, a normal completely positive subunital map $\mathcal{E}: \mathcal{B}(\mathcal{H}) \rightarrow \mathcal{B}(\mathcal{K})$, which is an arrow in Wstar ${ }_{\mathrm{CPSU}}$, corresponds to a completely positive trace-nonincreasing map $\mathcal{E}_{*}: \mathcal{T}(\mathcal{K}) \rightarrow \mathcal{T}(\mathcal{H})$, which is known as a quantum operation (see e.g. [32, §8.2], [17, Chap. 4]). This is the well-known duality between the Heisenberg and Schrödinger pictures: one transforms observables (i.e. self-adjoint operators), while another transforms states (i.e. density operators).

Hence, it is understood that our semantics for QPL by Wstar $\mathbf{C P S U}_{\text {is }}$ given in the Heisenberg picture, while Selinger's semantics by $\mathbf{Q}$ is given in the Schrödinger picture. In the words of [9], our semantics can also be thought of as the weakest precondition semantics. This is because a positive subunital map $\mathcal{E}: \mathcal{B}(\mathcal{H}) \rightarrow \mathcal{B}(\mathcal{K})$ can be restricted to a map $\mathcal{E}: \mathcal{E} f(\mathcal{H}) \rightarrow \mathcal{E} f(\mathcal{K})$ between their effects, where $\mathcal{E} f(\mathcal{H}):=$ $[0,1]_{\mathcal{B}(\mathcal{H})}$ is the set of effects on $\mathcal{H}$, and coincides with the set of predicates in [9].

\section{Embedding $\mathbf{Q}$ into $\left(\mathbf{W s t a r}_{\mathrm{CPSU}}\right)^{\mathrm{op}}$}

As seen in the previous section, the two semantics by $\mathbf{W s t a r}_{\mathrm{CPSU}}$ and $\mathbf{Q}$ can be considered as different viewpoints (Schrödinger vs. Heisenberg) for the same phenomena. We can state it categorically: the category $\mathbf{Q}$ can be contravariantly embedded into $\mathbf{W s t a r}_{\mathrm{CPSU}}$.

First we show the following embedding.

Theorem 8.1. There is a full embedding I: $\mathbf{C P M} \rightarrow\left(\mathbf{W s t a r}_{\mathrm{CP}}\right)^{\mathrm{op}}$.

Proof. Observe the following bijective correspondences.

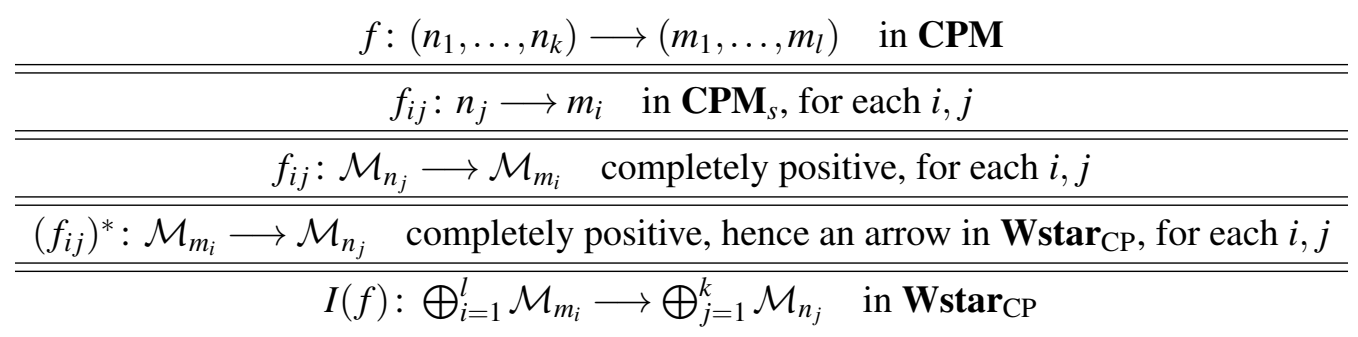

For the third correspondence, note that the self-duality of finite dimensional spaces:

$$
\mathcal{M}_{n_{j}} \cong \mathcal{B}\left(\mathbb{C}^{n_{j}}\right) \cong \mathcal{T}\left(\mathbb{C}^{n_{j}}\right)^{*} \cong\left(\mathcal{M}_{n_{j}}\right)^{*}
$$

The last correspondence comes from the fact finite direct sums are biproducts in Wstar $\mathbf{C P}_{\mathrm{CP}}$. Hence the mapping $I\left(n_{1}, \ldots, n_{k}\right)=\bigoplus_{j=1}^{k} \mathcal{M}_{n_{j}}$ defines a contravariant functor $I: \mathbf{C P M} \rightarrow\left(\mathbf{W s t a r}_{\mathrm{CP}}\right)^{\text {op }}$, which is full and faithful by definition, and clearly injective on objects.

We use the following lemma.

Lemma 8.2. Let $\mathcal{H}$ be a Hilbert space. A bounded operator $T \in \mathcal{B}(\mathcal{H})$ is positive if and only if $\operatorname{tr}(T S) \in$ $\mathbb{R}^{+}(=[0, \infty)$ ) for all positive $S \in \mathcal{T}(\mathcal{H})$.

Theorem 8.3. There is a full embedding $I^{\prime}: \mathbf{Q} \rightarrow\left(\mathbf{W s t a r}_{\mathrm{CPSU}}\right)^{\mathrm{op}}$. 
Proof. The functor $I: \mathbf{C P M} \rightarrow\left(\mathbf{W s t a r}_{\mathrm{CP}}\right)^{\text {op }}$ restricts to a full and faithful functor $I^{\prime}: \mathbf{Q} \rightarrow\left(\mathbf{W s t a r}_{\mathrm{CPSU}}\right)^{\mathrm{op}}$ as follows.

$$
\begin{aligned}
&f: \vec{n} \rightarrow \vec{m} \text { is trace-nonincreasing (Definition } 3.33) \\
& \Longleftrightarrow \sum_{i} \sum_{j} \operatorname{tr}\left(f_{i j}\left(A_{j}\right)\right) \leq \sum_{j} \operatorname{tr}\left(A_{j}\right) \text { for all }\left(A_{j}\right)_{j} \text { with positive } A_{j} \in \mathcal{M}_{n_{j}} \\
& \Longleftrightarrow \sum_{i} \operatorname{tr}\left(f_{i j}(A)\right) \leq \operatorname{tr}(A) \text { for each } A \in \mathcal{M}_{n_{j}}, \text { for each } j \\
& \Longleftrightarrow \sum_{i} \operatorname{tr}\left(\left(\left(f_{i j}\right)^{*}(1)\right) A\right) \leq \operatorname{tr}(A) \text { for each } A \in \mathcal{M}_{n_{j}}, \text { for each } j \\
& \Longleftrightarrow \operatorname{tr}\left(\left(1-\sum_{i}\left(f_{i j}\right)^{*}(1)\right) A\right) \geq 0 \text { for each } A \in \mathcal{M}_{n_{j}}, \text { for each } j \\
& \Longleftrightarrow 1-\sum_{i}\left(f_{i j}\right)^{*}(1) \geq 0 \text { for each } j \\
& \Longleftrightarrow \sum_{i}\left(f_{i j}\right)^{*}(1) \leq 1 \text { for each } j \\
& \Longleftrightarrow I(f)\left((1)_{i}\right) \leq(1)_{j} \\
& \Longleftrightarrow I(f): \bigoplus_{i} \mathcal{M}_{m_{i}} \longrightarrow \bigoplus_{j} \mathcal{M}_{n_{j}} \text { is subunital },
\end{aligned}
$$

where $\stackrel{\star}{\Longleftrightarrow}$ is by the equation (1) and $\stackrel{\star \star}{\Longleftrightarrow}$ is by Lemma 8.2 .

In fact, we can say more about the embedding. Notice that $I(\vec{n})$ is a finite dimensional $W^{*}$-algebra for each $\vec{n} \in \mathbf{C P M}$. Hence the embedding is restricted to $I_{\mathrm{fd}}: \mathbf{C P M} \rightarrow\left(\mathbf{F d W s t a r}_{\mathrm{CP}}\right)^{\text {op }}$, where $\mathbf{F d W s t a r}_{\mathrm{CP}}$ denotes the category of finite dimensional $W^{*}$-algebras and normal completely positive maps. In the same way we have an embedding $I_{\mathrm{fd}}^{\prime}: \mathbf{Q} \rightarrow\left(\mathbf{F d W s t a r}_{\mathrm{CPSU}}\right)^{\mathrm{op}}$.

Theorem 8.4. The embeddings

$$
I_{\mathrm{fd}}: \mathbf{C P M} \rightarrow\left(\text { FdWstar }_{\mathrm{CP}}\right)^{\mathrm{op}}, \quad I_{\mathrm{fd}}^{\prime}: \mathbf{Q} \rightarrow\left(\text { FdWstar }_{\mathrm{CPSU}}\right)^{\mathrm{op}}
$$

give equivalences of categories:

$$
\mathbf{C P M} \simeq\left(\mathbf{F d W s t a r}_{\mathrm{CP}}\right)^{\mathrm{op}}, \quad \mathbf{Q} \simeq\left(\mathbf{F d W s t a r}_{\mathrm{CPSU}}\right)^{\mathrm{op}} .
$$

Proof. [43, Theorem I.11.2] implies that $I_{\mathrm{fd}}$ and $I_{\mathrm{fd}}^{\prime}$ are essentially surjective. A full, faithful and essentially surjective functor is a part of equivalence [23, Theorem IV.4.1].

\section{Infinite types and classical computation}

Because Selinger's category $\mathbf{Q}$ is (contravariantly) embedded into $\mathbf{W} \operatorname{star}_{\mathrm{CPSU}}$, the category $\mathbf{W s t a \mathbf { C P S U } _ { \mathrm { C } }}$ can be thought of as an infinite dimensional extension of $\mathbf{Q}$. Therefore, working in the category $\mathbf{W s t a r}_{\mathrm{CPSU}}$ rather than $\mathbf{Q}$ enables us to handle infinite types.

Recall that a type bit is interpreted by $\llbracket$ bit $\rrbracket=\mathbb{C} \oplus \mathbb{C}$. It is easily extended to an interpretation of trit by $\llbracket$ trit $\rrbracket=\mathbb{C} \oplus \mathbb{C} \oplus \mathbb{C}$, and in general, an interpretations of a type of $n$-level classical system by $\bigoplus_{i=1}^{n} \mathbb{C}$. Then, it is natural to interpret a type nat of natural numbers by $\llbracket$ nat $\rrbracket=\bigoplus_{i \in \mathbb{N}} \mathbb{C}$, as Selinger also suggested in [39, §7.3]. Actually we have infinite direct sums in $\mathbf{W s t a r}_{\mathrm{CPSU}}$, but not in $\mathbf{Q}$.

We can also consider quantum analogue. Interpretations $\llbracket$ qbit $\rrbracket=\mathcal{M}_{2} \cong \mathcal{B}\left(\mathbb{C}^{2}\right), \llbracket \mathbf{q t r i t} \rrbracket=\mathcal{M}_{3} \cong$ $\mathcal{B}\left(\mathbb{C}^{3}\right), \ldots$, could be extended to an interpretation of a type of countable level quantum system (or "quantum natural numbers"?) by $\mathcal{B}\left(\mathcal{H}_{\mathbb{N}}\right)$, where $\mathcal{H}_{\mathbb{N}}$ is the Hilbert space of countable dimension. This type cannot be interpreted in $\mathbf{Q}$.

In what follows, we will generalise the former observation on classical types. 
Definition 9.1. For a set $S$ and for a real number $p \geq 1$, we define

$$
\ell^{p}(S):=\left\{\varphi:\left.S \rightarrow \mathbb{C}\left|\sum_{s \in S}\right| \varphi(s)\right|^{p}<\infty\right\}, \quad \ell^{\infty}(S):=\left\{\varphi: S \rightarrow \mathbb{C}\left|\sup _{s \in S}\right| \varphi(s) \mid<\infty\right\}
$$

It is a standard fact that these are Banach spaces with coordinatewise operations, and norms $\|\varphi\|_{p}=$ $\left(\sum_{s \in S}|\varphi(s)|^{p}\right)^{1 / p}$ and $\|\varphi\|_{\infty}=\sup _{s \in S}|\varphi(s)|$ respectively.

Then, notice that $\llbracket$ bit $\rrbracket=\mathbb{C} \oplus \mathbb{C} \cong \ell^{\infty}(2), \llbracket$ trit $\rrbracket=\mathbb{C} \oplus \mathbb{C} \oplus \mathbb{C} \cong \ell^{\infty}(3), \ldots, \llbracket$ nat $\rrbracket=\bigoplus_{i \in \mathbb{N}} \mathbb{C} \cong \ell^{\infty}(\mathbb{N})$. We have the following general result.

Proposition 9.2. Let $S$ and $T$ be sets.

1. $\ell^{\infty}(S)$ is a $W^{*}$-algebra with the predual $\ell^{1}(S)$.

2. Any function $f: S \rightarrow T$ induces a normal unital $*$-homomorphism $\ell^{\infty}(f): \ell^{\infty}(T) \rightarrow \ell^{\infty}(S)$ by $\ell^{\infty}(f)(\varphi)=\varphi \circ f$.

3. There is a (normal unital) $*$-isomorphism: $\ell^{\infty}(S) \bar{\otimes} \ell^{\infty}(T) \cong \ell^{\infty}(S \times T)$.

Proof. 1. It is straightforward to see $\ell^{\infty}(S)$ is a $C^{*}$-algebra. The duality $\ell^{\infty}(S) \cong \ell^{1}(S)^{*}$ is standard $4^{4}$

2. It is easy to see $\ell^{\infty}(f)$ is a unital $*$-homomorphism. To see it is normal, we can give explicitly the predual map $\ell^{1}(f): \ell^{1}(S) \rightarrow \ell^{1}(T)$ by $\ell^{1}(f)(\varphi)(t)=\sum_{s \in f^{-1}(t)} \varphi(s)$, which is bounded linear, and makes the following diagram commute.

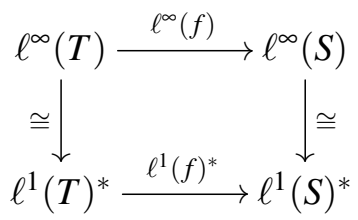

3. First we will show $\ell^{1}(S) \otimes \ell^{1}(S) \cong \ell^{1}(S \times T)$, i.e. the preduals are isometrically isomorphic. It is known that, when at least one of $W^{*}$-algebras $M, N$ is commutative, the dual spatial $C^{*}$-norm on $M_{*} \odot N_{*}$ coincides with the projective (i.e. greatest) cross norm [38, §1.22]. Hence $\ell^{1}(S) \otimes \ell^{1}(T)$ is the projective tensor product of $\ell^{1}(S)$ and $\ell^{1}(T)$, which possesses the universality to represent bounded bilinear maps $\ell^{1}(S) \times \ell^{1}(T) \rightarrow X$ (see [37, Theorem 2.9] for detail). Therefore, to prove $\ell^{1}(S) \otimes \ell^{1}(S) \cong \ell^{1}(S \times T)$, it suffices to show the following: there is a short bilinear map $\eta: \ell^{1}(S) \times \ell^{1}(T) \rightarrow \ell^{1}(S \times T)$ and for each short bilinear map $f: \ell^{1}(S) \times \ell^{1}(T) \rightarrow X$, there exists a unique short map $\bar{f}: \ell^{1}(S \times T) \rightarrow X$ such that $f=\bar{f} \circ \eta !^{5}$ We define $\eta(\varphi, \psi)(s, t)=\varphi(s) \psi(t)$, then it is easy to check $\eta$ is short bilinear. Let $f: \ell^{1}(S) \times \ell^{1}(T) \rightarrow X$ be a short bilinear map. Let

$$
F(S \times T)=\{\varphi: S \times T \rightarrow \mathbb{C} \mid \varphi \text { has finite support }\}
$$

be a vector space, which is a dense subspace of $\ell^{1}(S \times T)$. Note that $\{\delta(s, t) \mid s \in S, t \in T\}$ is a basis of this space, where $\delta: S \times T \rightarrow F(S \times T)$ is Kronecker's delta. Then we define a linear map $f^{\prime}: F(S \times T) \rightarrow X$

\footnotetext{
${ }^{4}$ A really standard result is the case where $S=\mathbb{N}$, but its proof is easily generalised. Alternatively, one can think of $\ell^{\infty}(S) \cong \ell^{1}(S)^{*}$ as a special case of $L^{\infty}(X, \Sigma, \mu) \cong L^{1}(X, \Sigma, \mu)^{*}$ for a localisable measure space $(X, \Sigma, \mu)$ (see e.g. [10 \$243]), using the counting measure on $S$.

${ }^{5}$ A bilinear map $f$ is short if $\|f(x, y)\| \leq\|x\|\|y\|$; and a linear map $g$ is short if $\|g(x)\| \leq\|x\|$.
} 
by $f^{\prime}(\delta(s, t))=f(\delta(s), \delta(t))$. With respect to $\ell^{1}$-norm, it is short because

$$
\begin{aligned}
\left\|f\left(\sum_{i=1}^{n} a_{i} \delta\left(s_{i}, t_{i}\right)\right)\right\| & =\left\|\sum_{i=1}^{n} a_{i} f\left(\delta\left(s_{i}\right), \delta\left(t_{i}\right)\right)\right\| \\
& \leq \sum_{i=1}^{n}\left|a_{i}\right|\left\|f\left(\delta\left(s_{i}\right), \delta\left(t_{i}\right)\right)\right\| \\
& \leq \sum_{i=1}^{n}\left|a_{i}\right|\left\|\delta\left(s_{i}\right)\right\|\left\|\delta\left(t_{i}\right)\right\| \\
& =\sum_{i=1}^{n}\left|a_{i}\right|=\left\|\sum_{i=1}^{n} a_{i} \delta\left(s_{i}, t_{i}\right)\right\| .
\end{aligned}
$$

Hence by density $f^{\prime}$ extends to a short map $\bar{f}: \ell^{1}(S \times T) \rightarrow X$. From $\bar{f}(\eta(\delta(s), \delta(t)))=\bar{f}(\delta(s, t))=$ $f(\delta(s), \delta(t))$, (bi)linearity and continuity, it follows that $\bar{f} \circ \eta=f$, and that such $\bar{f}$ is unique. We conclude that $\ell^{1}(S) \otimes \ell^{1}(T) \cong \ell^{1}(S \times T)$.

By dualising, we obtain an isomorphism

$$
\ell^{\infty}(S) \bar{\otimes} \ell^{\infty}(T) \cong\left(\ell^{1}(S) \otimes \ell^{1}(T)\right)^{*} \cong \ell^{1}(S \times T)^{*} \cong \ell^{\infty}(S \times T) .
$$

Finally we have to show this isomorphism is a $*$-isomorphism. Let $\Theta: \ell^{\infty}(S) \odot \ell^{\infty}(T) \rightarrow \ell^{\infty}(S \times T)$ be a map defined by $\Theta(\varphi \otimes \psi)(s, t)=\varphi(s) \varphi(t)$. Then it is easy to check $\Theta$ is a $*$-homomorphism. Now it suffices to show the following diagram commutes,

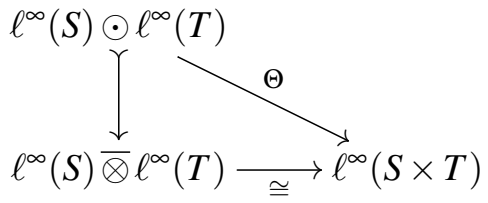

because the canonical embedding $\ell^{\infty}(S) \odot \ell^{\infty}(T) \rightarrow \ell^{\infty}(S) \bar{\otimes} \ell^{\infty}(T)$ is ultraweakly dense. Checking this commutativity is straightforward.

Corollary 9.3. There is an embedding $\ell^{\infty}$ : Set $\rightarrow\left(\mathbf{C W s t a r}_{\mathrm{MIU}}\right)^{\mathrm{op}}$, from the category of sets and functions to the category of commutative $W^{*}$-algebras and unital $*$-homomorphisms. Moreover, it maps binary product of sets to the spatial $W^{*}$-tensor product.

Proof. By Proposition 9.2 , $\ell^{\infty}$ defines a functor which maps binary product of sets to the spatial $W^{*}$ tensor product. The injectivity on objects is obvious. We show it is faithful. Let $f, g: S \rightarrow T$ be functions with $\ell^{\infty}(f)=\ell^{\infty}(g)$. Let $\delta: T \rightarrow \ell^{\infty}(T)$ be Kronecker's delta. Then, for each $s \in S$,

$$
\delta(f(s))(g(s))=\ell^{\infty}(g)(\delta(f(s)))(s)=\ell^{\infty}(f)(\delta(f(s)))(s)=\delta(f(s))(f(s))=1,
$$

so that $f(s)=g(s)$. Hence $f=g$.

As a consequence, we can embed sets $S_{1}, \ldots, S_{n}, T$ and a function $f: S_{1} \times \cdots \times S_{n} \rightarrow T$ contravariantly into the category $\mathbf{C W s t a r}_{\mathrm{MIU}}$ (hence into $\mathbf{W s t a r}_{\mathrm{CPSU}}$ ) as:

$$
\ell^{\infty}(f): \ell^{\infty}(T) \longrightarrow \ell^{\infty}\left(S_{1} \times \cdots \times S_{n}\right) \cong \ell^{\infty}\left(S_{1}\right) \bar{\otimes} \cdots \bar{\otimes} \ell^{\infty}\left(S_{n}\right)
$$

Therefore, any classical data type and function between them interpreted in Set can also be interpreted

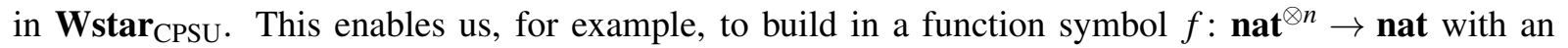
(classical) interpretation $\llbracket f \rrbracket: \mathbb{N}^{n} \rightarrow \mathbb{N}$ into a quantum programming language. 


\section{Conclusions and future work}

We have given a novel denotational semantics for a first-order functional quantum programming language QPL by operator algebras. Technically, we showed that the category Wstar $\mathbf{C P S U}_{\text {of }} W^{*}$-algebras and normal completely positive subunital maps is a Dcppo ${ }_{\perp}$-enriched symmetric monoidal category with Dcppo $_{\perp}$-enriched finite products, and therefore its opposite is an elementary quantum flow chart category. Our model can be considered as an infinite dimensional extension of Selinger's original model $\mathbf{Q}$, and is more flexible model to accommodate infinite structures and to unify classical computation as commutative structures.

In parallel with the present work, Rennela [36] recently showed that Wstar PSU $_{\text {is }}$ is algebraically compact for a certain class of ("von Neumann") functors. These results demonstrate that the use of operator algebras, especially $W^{*}$-algebras, for modelling quantum computation is quite promising. One of the most important future work is to give a denotational semantics by operator algebras for a higher-order quantum programming language, or the quantum lambda calculus. In an (unpublished) paper [21], it is showed that the symmetric monoidal category $\left(\left(\mathbf{W s t a r}_{\mathrm{MIU}}\right)^{\mathrm{op}}, \bar{\otimes}, \mathbb{C}\right)$ is closed. This result can be helpful.

Another future work is, extending the results in $\$ 9$, to study commutative $W^{*}$-algebras for modelling classical computation. It is known that commutative $W^{*}$-algebras are characterised as $L^{\infty}$ spaces $L^{\infty}(X, \Sigma, \mu)$ of localisable measure spaces $(X, \Sigma, \mu)$ (see e.g. [38, §1.18]). Hence, by analogy with the categorical (Gelfand) duality between commutative $C^{*}$-algebras and compact Hausdorff spaces, it is natural to expect the categorical duality between commutative $W^{*}$-algebras and certain measure (or measurable) spaces. In that case, Proposition 9.2 and Corollary 9.3 could be obtained as a special case for counting measures. We further expect a similar result to [11], showing that $\mathbf{W s t a r}_{\mathrm{CPSU}}$ can also accommodate probabilistic computation.

\section{Acknowledgements}

This paper is based on my master thesis [6] at the University of Tokyo. I am deeply grateful to my supervisor Ichiro Hasuo and colleagues at the time for valuable discussions. I also wish to thank my current colleagues at Radboud University Nijmegen and the QPL reviewers for helpful comments.

\section{References}

[1] Samson Abramsky \& Achim Jung (1994): Domain Theory. In: Handbook of Logic in Computer Science, III, Clarendon Press, pp. 1-168. Corrected and expanded version available online.

[2] Huzihiro Araki (1999): Mathematical Theory of Quantum Fields. International Series of Monographs on Physics 101, Oxford University Press. Originally published in Japanese as Ryoshiba no Suri (Iwanami Shoten, 1993).

[3] Ola Bratteli \& Derek W. Robinson (1987/1997): Operator Algebras and Quantum Statistical Mechanics (2 volumes), second edition. Texts and Monographs in Physics, Springer, doi:10.1007/978-3-662-02520-8 and 10.1007/978-3-662-03444-6.

[4] Nathanial P. Brown \& Narutaka Ozawa (2008): $C^{*}$-Algebras and Finite-Dimensional Approximations. Graduate Studies in Mathematics 88, American Mathematical Society.

[5] Giulio Chiribella, Alessandro Toigo \& Veronica Umanità (2013): Normal Completely Positive Maps on the Space of Quantum Operations. Open Systems \& Information Dynamics 20(1), doi:10.1142/ S1230161213500030 
[6] Kenta Cho (2014): Semantics for a Quantum Programming Language by Operator Algebras. Master's thesis, The University of Tokyo. Available at http://www-mmm.is.s.u-tokyo.ac.jp/ ₹ckn/papers/ master-thesis.pdf.

[7] John B. Conway (2000): A Course in Operator Theory. Graduate Studies in Mathematics 21, American Mathematical Society.

[8] Giacomo Mauro D’Ariano, Dennis Kretschmann, Dirk Schlingemann \& Reinhard F. Werner (2007): Reexamination of quantum bit commitment: The possible and the impossible. Phys. Rev. A 76(032328), doi $10.1103 /$ PhysRevA.76.032328.

[9] Ellie D'Hondt \& Prakash Panangaden (2006): Quantum weakest preconditions. Mathematical Structures in Computer Science 16, pp. 429-451, doi:10.1017/S0960129506005251.

[10] D. H. Fremlin (2010): Measure Theory, Volume 2, second edition. Torres Fremlin.

[11] Robert Furber \& Bart Jacobs (2013): From Kleisli Categories to Commutative C*-Algebras: Probabilistic Gelfand Duality. In: Algebra and Coalgebra in Computer Science (CALCO) 2013, Lecture Notes in Computer Science 8089, Springer, pp. 141-157, doi:10.1007/978-3-642-40206-7_12.

[12] Simon J. Gay (2006): Quantum programming languages: survey and bibliography. Mathematical Structures in Computer Science 16, pp. 581-600, doi $10.1017 /$ S0960129506005378

[13] Rudolf Haag (1996): Local Quantum Physics: Fields, Particles, Algebras, second edition. Theoretical and Mathematical Physics, Springer, doi:10.1007/978-3-642-61458-3.

[14] Rudolf Haag \& Daniel Kastler (1964): An Algebraic Approach to Quantum Field Theory. Journal of Mathematical Physics 5(7), pp. 848-861, doi:10.1063/1.1704187.

[15] Masahito Hasegawa (1999): Models of Sharing Graphs: A Categorical Semantics of let and letrec. Distinguished Dissertations, Springer, doi:10.1007/978-1-4471-0865-8

[16] Ichiro Hasuo \& Naohiko Hoshino (2011): Semantics of Higher-Order Quantum Computation via Geometry of Interaction. In: The 26th Annual IEEE Symposium on Logic in Computer Science (LICS 2011), doi:10. 1109/LICS.2011.26.

[17] Teiko Heinosaari \& Mário Ziman (2012): The Mathematical Language of Quantum Theory: From Uncertainty to Entanglement. Cambridge University Press.

[18] Naohiko Hoshino, Koko Muroya \& Ichiro Hasuo (2014): Memoryful Geometry of Interaction: From Coalgebraic Components to Algebraic Effects. In: Joint Meeting of the Twenty-Third EACSL Annual Conference on Computer Science Logic and the Twenty-Ninth Annual ACM/IEEE Symposium on Logic in Computer Science (CSL-LICS 2014), doi:10.1145/2603088.2603124. Extended version with appendix is available at the first author's website.

[19] Bart Jacobs (2013): On Block Structures in Quantum Computation. Electronic Notes in Theoretical Computer Science 298, pp. 233-255, doi $10.1016 / \mathrm{j}$.entcs.2013.09.016.

[20] Michael Keyl (2002): Fundamentals of quantum information theory. Physics Reports 369(5), pp. 431-548, doi $10.1016 / \mathrm{S} 0370-1573(02) 00266-1$.

[21] Andre Kornell (2012): Quantum Collections. ArXiv:1202.2994v1 [math.OA].

[22] Nicolaas P. Landsman (2009): Algebraic Quantum Mechanics. In: Compendium of Quantum Physics, Springer, pp. 6-10, doi:10.1007/978-3-540-70626-7_3

[23] Saunders Mac Lane (1998): Categories for the Working Mathematician, second edition. Graduate Texts in Mathematics 5, Springer.

[24] Octavio Malherbe, Philip Scott \& Peter Selinger (2013): Presheaf Models of Quantum Computation: An Outline. In: Computation, Logic, Games, and Quantum Foundations. The Many Facets of Samson Abramsky, Lecture Notes in Computer Science 7860, Springer, pp. 178-194, doi:10.1007/978-3-642-38164-5_13.

[25] Ralf Meyer (2008): Categorical aspects of bivariant K-theory. In: K-Theory and Noncommutative Geometry, EMS Series of Congress Reports, European Mathematical Society, pp. 1-39, doi:10.4171/060-1/1. ArXiv:math/0702145 [math.KT]. 
[26] Francis J. Murray \& John von Neumann (1936): On Rings of Operators. Annals of Mathematics 37(1), pp. 116-229, doi:10.2307/1968693.

[27] Francis J. Murray \& John von Neumann (1937): On Rings of Operators. II. Transactions of the American Mathematical Society 41(2), pp. 208-248, doi:10.1090/S0002-9947-1937-1501899-4

[28] Francis J. Murray \& John von Neumann (1943): On Rings of Operators. IV. Annals of Mathematics 44(4), pp. 716-808, doi: $10.2307 / 1969107$.

[29] John von Neumann (1940): On Rings of Operators. III. Annals of Mathematics 41(1), pp. 94-161, doi:10. $2307 / 1968823$

[30] John von Neumann (1949): On Rings of Operators. Reduction Theory. Annals of Mathematics 50(2), pp. 401-485, doi:10.2307/1969463.

[31] John von Neumann (1955): Mathematical Foundations of Quantum Mechanics. Princeton University Press. Originally published in German as Mathematische Grundlagen der Quantenmechanik (Springer, 1932).

[32] Michael A. Nielsen \& Isaac L. Chuang (2000): Quantum Computation and Quantum Information. Cambridge University Press.

[33] Michele Pagani, Peter Selinger \& Benoît Valiron (2014): Applying Quantitative Semantics to Higher-order Quantum Computing. In: The 41st ACM SIGPLAN-SIGACT Symposium on Principles of Programming Languages (POPL 2014), doi:10.1145/2535838.2535879.

[34] Miklós Rédei (1996): Why John von Neumann did not Like the Hilbert Space formalism of quantum mechanics (and what he liked instead). Studies in History and Philosophy of Science Part B: Studies in History and Philosophy of Modern Physics 27(4), pp. 493-510, doi:10.1016/S1355-2198(96)00017-2.

[35] Mathys Rennela (2013): On operator algebras in quantum computation. Master's thesis, Université Paris 7 Denis Diderot. Available at http://www.cs.ru.nl/ mathysr/papers/masterthesis.pdf.

[36] Mathys Rennela (2014): Towards a Quantum Domain Theory: Order-Enrichment and Fixpoints in $W^{*-}$ Algebras. In: The Thirtieth Conference on the Mathematical Foundations of Programming Semantics (MFPS $X X X)$. To appear.

[37] Raymond A. Ryan (2002): Introduction to Tensor Products of Banach Spaces. Springer Monographs in Mathematics, Springer, doi $10.1007 / 978-1-4471-3903-4$

[38] Shôichirô Sakai (1998): $C^{*}$-Algebras and $W^{*}$-Algebras. Classics in Mathematics, Springer. Reprint of the 1971 Edition.

[39] Peter Selinger (2004): Towards a quantum programming language. Mathematical Structures in Computer Science 14, pp. 527-586, doi:10.1017/S0960129504004256.

[40] Peter Selinger \& Benoît Valiron (2006): A lambda calculus for quantum computation with classical control. Mathematical Structures in Computer Science 16, pp. 527-552, doi:10.1017/S0960129506005238.

[41] Peter Selinger \& Benoît Valiron (2008): On a Fully Abstract Model for a Quantum Linear Functional Language: (Extended Abstract). Electronic Notes in Theoretical Computer Science 210(0), pp. 123-137, doi $10.1016 /$ j.entcs.2008.04.022

[42] Peter Selinger \& Benoît Valiron (2009): Quantum lambda calculus. In Simon Gay \& Ian Mackie, editors: Semantic Techniques in Quantum Computation, Cambridge University Press, pp. 135-172, doi:10.1017/ CBO9781139193313.005.

[43] Masamichi Takesaki (2001/2003): Theory of Operator Algebras (3 volumes). Encyclopaedia of Mathematical Sciences 124-125, 127, Springer.

[44] Benoît Valiron (2013): Quantum Computation: From a Programmer's Perspective. New Generation Computing 31(1), pp. 1-26, doi:10.1007/s00354-012-0120-0. 


\section{A Monoidal traces on $\omega$ Cppo-enriched cartesian categories}

Here we will give a proof of Theorem 3.4. We in fact show the dual statement, i.e. Theorem A.1 and Theorem A.4 below, because cartesian categories are more standard than cocartesian categories.

First, let us clarify terminology here. A cartesian category is a (symmetric) monoidal category whose monoidal structure is given by finite products. In other words, it is just a category with (a choice of) finite products. A functor between cartesian categories is cartesian if it preserves finite products. Note that the

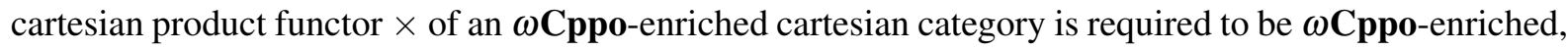
or equivalently, the tupling $\langle\cdot, \cdot\rangle$ be $\omega$-continuous.

The following is the first theorem we wish to prove.

Theorem A.1. Every $\omega$ Cppo-enriched cartesian category with left-strict composition (i.e. $\perp \circ f=\perp$ ) is traced. For $f: A \times X \rightarrow B \times X$, the trace $\operatorname{Tr}(f): A \rightarrow B$ is given by

$$
\operatorname{Tr}(f):=\pi_{1} \circ \bigvee_{n \in \mathbb{N}} \operatorname{Tr}^{(n)}(f)
$$

where $\operatorname{Tr}^{(n)}(f): A \rightarrow B \times X$ is defined by

$$
\begin{aligned}
\operatorname{Tr}^{(0)}(f) & =\perp \\
\operatorname{Tr}^{(n+1)}(f) & =f \circ\left\langle\mathrm{id}_{A}, \pi_{2} \circ \operatorname{Tr}^{(n)}(f)\right\rangle .
\end{aligned}
$$

We use the well-known theorem of Hasegawa and Hyland. The complete proof is found in Hasegawa's thesis [15, Theorem 7.1.1].

Theorem A.2 (Hasegawa/Hyland). A cartesian category is traced if and only if it has a Conway operator. A trace operator $\operatorname{Tr}$ and a Conway operator Fix are related bijectively as follows:

$$
\operatorname{Tr}(f)=\pi_{1} \circ f \circ\left\langle\mathrm{id}_{A}, \operatorname{Fix}\left(\pi_{2} \circ f\right)\right\rangle
$$

for $f: A \times X \rightarrow B \times X$, and

$$
\operatorname{Fix}(g)=\operatorname{Tr}\left(\Delta_{X} \circ g\right)
$$

for $g: A \times X \rightarrow X$, where $\Delta_{X}=\left\langle\mathrm{id}_{X}, \mathrm{id}_{X}\right\rangle$ is the diagonal map.

Thanks to this theorem, the problem is reduced to a little easier problem on a Conway operator. The following result is already showed in [18, Lemma A.1 in Appendix].

Theorem A.3. Every $\omega$ Cppo-enriched cartesian category with left-strict composition (i.e. $\perp \circ f=\perp$ ) has a Conway operator Fix. For $g: A \times X \rightarrow X, \operatorname{Fix}(g): A \rightarrow X$ is given by

$$
\operatorname{Fix}(g):=\bigvee_{n \in \mathbb{N}} \operatorname{Fix}^{(n)}(g)
$$

where $\operatorname{Fix}^{(n)}(g): A \rightarrow X$ is defined by

$$
\begin{aligned}
\operatorname{Fix}^{(0)}(g) & =\perp \\
\operatorname{Fix}^{(n+1)}(g) & =g \circ\left\langle\operatorname{id}_{A}, \operatorname{Fix}^{(n)}(g)\right\rangle .
\end{aligned}
$$

Now we prove Theorem A.1 as follows. 
Proof of Theorem A.1 Let $\mathbf{C}$ be an $\omega$ Cppo-enriched cartesian category with left-strict composition. Then, by Theorem A.2 and Theorem A.3, C is traced. We still need to check the equation (2). For $f: A \times X \rightarrow B \times X$,

$$
\begin{array}{rlrl}
\operatorname{Tr}(f) & =\pi_{1} \circ f \circ\left\langle\operatorname{id}_{A}, \operatorname{Fix}\left(\pi_{2} \circ f\right)\right\rangle & & \text { by (3) } \\
& =\pi_{1} \circ f \circ\left\langle\operatorname{id}_{A}, \bigvee_{n \in \mathbb{N}} \operatorname{Fix}^{(n)}\left(\pi_{2} \circ f\right)\right\rangle & & \text { by (4) } \\
& =\pi_{1} \circ \bigvee_{n \in \mathbb{N}}\left(f \circ\left\langle\operatorname{id}_{A}, \operatorname{Fix}^{(n)}\left(\pi_{2} \circ f\right)\right\rangle\right) . &
\end{array}
$$

It is easy to see, by induction on $n$,

$$
\operatorname{Tr}^{(n)}(f) \leq f \circ\left\langle\operatorname{id}_{A}, \operatorname{Fix}^{(n)}\left(\pi_{2} \circ f\right)\right\rangle \leq \operatorname{Tr}^{(n+1)}(f)
$$

for all $n \in \mathbb{N}$. It follows that

$$
\bigvee_{n \in \mathbb{N}}\left(f \circ\left\langle\operatorname{id}_{A}, \operatorname{Fix}^{(n)}\left(\pi_{2} \circ f\right)\right\rangle\right)=\bigvee_{n \in \mathbb{N}} \operatorname{Tr}^{(n)}(f) .
$$

Hence we have

$$
\operatorname{Tr}(f)=\pi_{1} \circ \bigvee_{n \in \mathbb{N}} \operatorname{Tr}^{(n)}(f) .
$$

Next, we will show the theorem on cartesian functors.

Theorem A.4. Let $\mathbf{C}$ and $\mathbf{D}$ be $\omega \mathbf{C p p o - e n r i c h e d ~ c a r t e s i a n ~ c a t e g o r i e s , ~ w h i c h ~ a r e ~ t r a c e d ~ b y ~ T h e o r e m ~ A . 1 ~}$ Then, every $\omega \mathbf{C p p o - e n r i c h e d ~ c a r t e s i a n ~ f u n c t o r ~ b e t w e e n ~} \mathbf{C}$ and $\mathbf{D}$ satisfying $F \perp=\perp$ is traced.

Proof. Specifically, we need to show the equation

$$
F \operatorname{Tr}(f)=\operatorname{Tr}\left(\phi_{B} \circ F f \circ \phi_{A}^{-1}\right)
$$

for $f: A \times X \rightarrow B \times X$ in $\mathbf{C}$, where

$$
\begin{aligned}
& \phi_{A}:=\left\langle F \pi_{1}, F \pi_{2}\right\rangle: F(A \times X) \stackrel{\cong}{\longrightarrow} F A \times F X \\
& \phi_{B}:=\left\langle F \pi_{1}, F \pi_{2}\right\rangle: F(B \times X) \stackrel{\cong}{\longrightarrow} F B \times F X
\end{aligned}
$$

are the canonical isomorphisms. Note first that we have the following commutative diagrams.
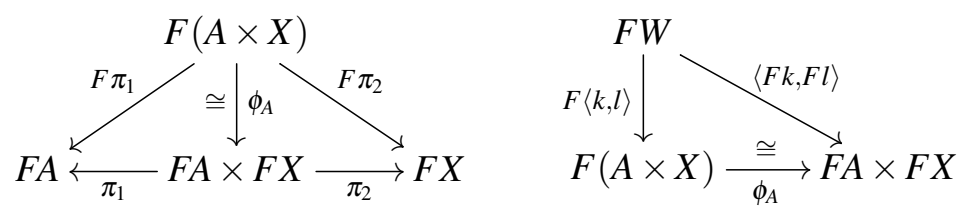

In the right diagram, $k: W \rightarrow A$ and $l: W \rightarrow X$ are arbitrary arrows in $\mathbf{C}$. Similar diagrams commute for $\phi_{B}$. Then,

$$
\begin{aligned}
F \operatorname{Tr}(f) & =F\left(\pi_{1} \circ \bigvee_{n \in \mathbb{N}} \operatorname{Tr}^{(n)}(f)\right) \\
& =F \pi_{1} \circ \bigvee_{n \in \mathbb{N}} F \operatorname{Tr}^{(n)}(f) \\
& =\pi_{1} \circ \phi_{B} \circ \bigvee_{n \in \mathbb{N}} F \operatorname{Tr}^{(n)}(f) \\
& =\pi_{1} \circ \bigvee_{n \in \mathbb{N}}\left(\phi_{B} \circ F \operatorname{Tr}^{(n)}(f)\right),
\end{aligned}
$$


while

$$
\operatorname{Tr}\left(\phi_{B} \circ F f \circ \phi_{A}^{-1}\right)=\pi_{1} \circ \bigvee_{n \in \mathbb{N}} \operatorname{Tr}^{(n)}\left(\phi_{B} \circ F f \circ \phi_{A}^{-1}\right) .
$$

Hence it suffices to show

$$
\bigvee_{n \in \mathbb{N}}\left(\phi_{B} \circ F \operatorname{Tr}^{(n)}(f)\right)=\bigvee_{n \in \mathbb{N}} \operatorname{Tr}^{(n)}\left(\phi_{B} \circ F f \circ \phi_{A}^{-1}\right)
$$

This follows from

$$
\operatorname{Tr}^{(n)}\left(\phi_{B} \circ F f \circ \phi_{A}^{-1}\right) \leq \phi_{B} \circ F \operatorname{Tr}^{(n)}(f) \leq \operatorname{Tr}^{(n+1)}\left(\phi_{B} \circ F f \circ \phi_{A}^{-1}\right)
$$

for all $n \in \mathbb{N}$. We will prove it by induction on $n$.

(i) Base case $(n=0)$ :

$$
\operatorname{Tr}^{(0)}\left(\phi_{B} \circ F f \circ \phi_{A}^{-1}\right)=\perp \leq \phi_{B} \circ F \operatorname{Tr}^{(0)}(f)
$$

shows the first inequality, and

$$
\begin{aligned}
& \operatorname{Tr}^{(1)}\left(\phi_{B} \circ F f \circ \phi_{A}^{-1}\right) \\
& =\phi_{B} \circ F f \circ \phi_{A}^{-1} \circ\left\langle\mathrm{id}_{F A}, \pi_{2} \circ \operatorname{Tr}^{(0)}\left(\phi_{B} \circ F f \circ \phi_{A}^{-1}\right)\right\rangle \\
& \geq \phi_{B} \circ \perp \\
& =\phi_{B} \circ F \perp \\
& =\phi_{B} \circ F \operatorname{Tr}^{(0)}(f)
\end{aligned}
$$

shows the second inequality.

(ii) Induction step:

$$
\begin{aligned}
& \phi_{B} \circ F \operatorname{Tr}^{(n+1)}(f) \\
& =\phi_{B} \circ F\left(f \circ\left\langle\operatorname{id}_{A}, \pi_{2} \circ \operatorname{Tr}^{(n)}(f)\right\rangle\right. \\
& =\phi_{B} \circ F f \circ \phi_{A}^{-1} \circ\left\langle F \operatorname{Fid}_{A}, F\left(\pi_{2} \circ \operatorname{Tr}^{(n)}(f)\right)\right\rangle \\
& =\phi_{B} \circ F f \circ \phi_{A}^{-1} \circ\left\langle\operatorname{id}_{F A}, \pi_{2} \circ \phi_{B} \circ \operatorname{Tr}^{(n)}(f)\right\rangle \\
& \geq \phi_{B} \circ F f \circ \phi_{A}^{-1} \circ\left\langle\operatorname{id}_{F A}, \pi_{2} \circ \operatorname{Tr}^{(n)}\left(\phi_{B} \circ F f \circ \phi_{A}^{-1}\right)\right\rangle \\
& =\operatorname{Tr}^{(n+1)}\left(\phi_{B} \circ F f \circ \phi_{A}^{-1}\right)
\end{aligned}
$$

by I.H.

shows the first inequality, and similarly

$$
\begin{aligned}
& \phi_{B} \circ F \operatorname{Tr}^{(n+1)}(f) \\
& =\phi_{B} \circ F f \circ \phi_{A}^{-1} \circ\left\langle\operatorname{id}_{F A}, \pi_{2} \circ \phi_{B} \circ \operatorname{Tr}^{(n)}(f)\right\rangle \\
& \leq \phi_{B} \circ F f \circ \phi_{A}^{-1} \circ\left\langle\operatorname{id}_{F A}, \pi_{2} \circ \operatorname{Tr}^{(n+1)}\left(\phi_{B} \circ F f \circ \phi_{A}^{-1}\right)\right\rangle \\
& =\operatorname{Tr}^{(n+2)}\left(\phi_{B} \circ F f \circ \phi_{A}^{-1}\right)
\end{aligned}
$$

by I.H.

shows the second inequality.

Therefore we have

$$
F \operatorname{Tr}(f)=\operatorname{Tr}\left(\phi_{B} \circ F f \circ \phi_{A}^{-1}\right) .
$$




\section{B Distribution of tensor products over direct sums}

First we recap tensor products of $C^{*}$ - and $W^{*}$-algebras. Let $A$ and $B$ be $C^{*}$-algebras. We denote by $A \odot B$ the algebraic tensor product of $A$ and $B$. Then it is easy to see $A \odot B$ is a $*$-algebra. A $C^{*}$-norm on $A \odot B$ is not necessarily unique, but there exists a least $C^{*}$-norm on $A \odot B$, which is called the spatial (or minimal, or injective) $C^{*}$-norm. The spatial (or minimal, or injective) $C^{*}$-tensor product $A \otimes B$ of $A$ and $B$ is defined to be the completion of $A \odot B$ under the spatial $C^{*}$-norm. Later we will use the next result, which also explains the term "spatial".

Theorem B.1 ([43, Theorem IV.4.9.(iii)]). Let $A, B$ be $C^{*}$-algebras, and $\left(\mathcal{H}_{A}, \pi_{A}\right),\left(\mathcal{H}_{B}, \pi_{B}\right)$ faithful representations of $A, B$ respectively. Then the mapping $a \otimes b \mapsto \pi_{A}(a) \otimes \pi_{B}(b)$ extends to a faithful representation $\pi_{A} \otimes \pi_{B}: A \otimes B \rightarrow \mathcal{B}\left(\mathcal{H}_{A} \otimes \mathcal{H}_{B}\right)$ of $A \otimes B$ on $\mathcal{H}_{A} \otimes \mathcal{H}_{B}$.

We also use a similar (but much easier to prove) result on direct sums.

Theorem B.2. Let $A, B$ be $C^{*}$-algebras, and $\left(\mathcal{H}_{A}, \pi_{A}\right),\left(\mathcal{H}_{B}, \pi_{B}\right)$ faithful representations of $A, B$ respectively. Then the mapping $(a, b) \mapsto \pi_{A}(a) \oplus \pi_{B}(b)$ gives a faithful representation $\pi_{A} \oplus \pi_{B}: A \oplus B \rightarrow$ $\mathcal{B}\left(\mathcal{H}_{A} \oplus \mathcal{H}_{B}\right)$ of $A \oplus B$ on $\mathcal{H}_{A} \oplus \mathcal{H}_{B}$.

Let $M$ and $N$ be $W^{*}$-algebras. Consider the algebraic tensor product $M_{*} \odot N_{*}$ of their preduals $M_{*}$ and $N_{*}$. Because the spatial $C^{*}$-norm is a cross norm, we can equip $M_{*} \odot N_{*}$ with the dual norm of the spatial $C^{*}$-norm via the following embedding:

$$
M_{*} \odot N_{*} \hookrightarrow M^{*} \odot N^{*} \hookrightarrow(M \otimes N)^{*} .
$$

Let $M_{*} \otimes N_{*}$ denote the completion of $M_{*} \odot N_{*}$ under this norm. Then, it turns out that the dual $\left(M_{*} \otimes N_{*}\right)^{*}$ is a $C^{*}$-algebra, hence a $W^{*}$-algebra with the predual $M_{*} \otimes N_{*}$. The $W^{*}$-algebra $\left(M_{*} \otimes N_{*}\right)^{*}$ is denoted by $M \bar{\otimes} N$, and called the spatial $W^{*}$-tensor product of $M$ and $N$. The following fact is useful: the spatial $C^{*}$-tensor product $M \otimes N$ is ultraweakly densely embedded into $M \bar{\otimes} N$ (and therefore $M \odot N$ also forms a dense subspace of $M \bar{\otimes} N$ ). With respect to this canonical embeddings, it is straightforward to see that the $C^{*}$-tensor product of maps $f \otimes g$ (if defined) extends to the $W^{*}$-tensor product $f \bar{\otimes} g=\left(f_{*} \otimes g_{*}\right)^{*}$. For further details of tensor products of $C^{*}$ - and $W^{*}$-algebras, see e.g. [38, §1.22], [43, Chapter IV].

For $p$ with $1 \leq p \leq \infty$, we will denote by $\oplus^{p}$ the $\ell^{p}$-direct sum of normed spaces. Note that the direct sum of $C^{*}$ - and $W^{*}$-algebras is the $\ell^{\infty}$-direct sum. For $W^{*}$-algebras $\left(M_{i}\right)_{i}$, the predual of $\bigoplus_{i} M_{i}$ is given by $\bigoplus_{i}^{1} M_{i *}$, i.e. $\left(\bigoplus_{i}^{1} M_{i *}\right)^{*} \cong \bigoplus_{i} M_{i}$ ([38. Definition 1.1.5]). For the finite direct sum, we have the other result.

Lemma B.3. Let $X, Y$ be normed spaces. Then we have an isometric isomorphism:

$$
\left(X \oplus^{\infty} Y\right)^{*} \cong X^{*} \oplus^{1} Y^{*}
$$

We will also use the following elementary result.

Lemma B.4. Let $f: X \rightarrow X^{\prime}$ and $g: Y \rightarrow Y^{\prime}$ be isometries with dense images between normed spaces. For any $p(1 \leq p \leq \infty), f \oplus^{p} g: X \oplus^{p} Y \rightarrow X^{\prime} \oplus^{p} Y^{\prime}$ is an isometry with dense image, too.

Now, we prove the distribution of $C^{*}$ - and $W^{*}$-tensor products over direct sums.

Theorem B.5. Let $A, B, C$ be $C^{*}$-algebras. Then the canonical maps

$$
\begin{gathered}
!: A \otimes 0 \longrightarrow 0 \\
\left\langle\mathrm{id} \otimes \pi_{1}, \mathrm{id} \otimes \pi_{2}\right\rangle: A \otimes(B \oplus C) \longrightarrow(A \otimes B) \oplus(A \otimes C)
\end{gathered}
$$

are $*$-isomorphisms. 
Proof. Note that $A \odot 0 \cong 0$ and the only possible norm is the trivial one. Therefore $A \otimes 0 \cong A \odot 0 \cong 0$ and the first one is proved.

We will show the latter one. Let

$$
\begin{aligned}
& \iota_{1}: A \odot(B \oplus C) \hookrightarrow A \otimes(B \oplus C) \\
& l_{2}: A \odot B \hookrightarrow A \otimes B \\
& \iota_{3}: A \odot C \hookrightarrow A \otimes C
\end{aligned}
$$

be the dense embeddings. Note that the algebraic tensor product distributes over finite direct sum, and that the following diagram commutes.

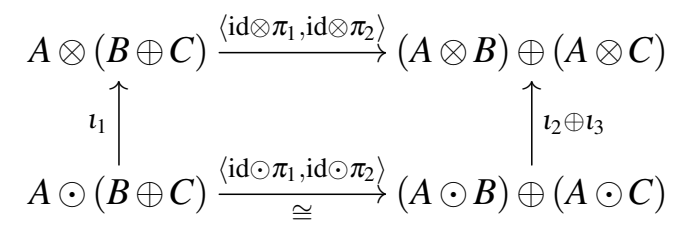

By the universality of completion, it suffices to show $\left(\boldsymbol{l}_{B} \oplus \boldsymbol{l}_{C}\right) \circ\left\langle\mathrm{id} \odot \pi_{1}, \mathrm{id} \odot \boldsymbol{\pi}_{2}\right\rangle$ is an isometry with dense image w.r.t. the spatial $C^{*}$-norm on $A \odot(B \oplus C)$. By Lemma B.4, $\boldsymbol{l}_{B} \oplus \boldsymbol{l}_{C}$ is an isometry with dense image w.r.t. the spatial $C^{*}$-norms on $A \odot B$ and $A \odot C$. Hence, we only need to prove $\left\langle\operatorname{id} \odot \pi_{1}, \operatorname{id} \odot \pi_{2}\right\rangle$ is an isometry. Let $\left(\mathcal{H}_{A}, \pi_{A}\right),\left(\mathcal{H}_{B}, \pi_{B}\right),\left(\mathcal{H}_{C}, \pi_{C}\right)$ be faithful representations of $A, B, C$ respectively. Then we have a faithful representation $\left(\mathcal{H}_{B} \oplus \mathcal{H}_{C}, \pi_{B} \oplus \pi_{C}\right)$ of $B \oplus C$, and a faithful representation $\left(\mathcal{H}_{A} \otimes\left(\mathcal{H}_{B} \oplus\right.\right.$ $\left.\left.\mathcal{H}_{C}\right), \pi_{A} \otimes\left(\pi_{B} \oplus \pi_{C}\right)\right)$ of $A \otimes(B \oplus C)$. On the other hand, $\left(\mathcal{H}_{A} \otimes \mathcal{H}_{B}, \pi_{A} \otimes \pi_{B}\right)$ and $\left(\mathcal{H}_{A} \otimes \mathcal{H}_{C}, \pi_{A} \otimes \pi_{C}\right)$ is faithful representations of $A \otimes B$ and $A \otimes C$ respectively, and hence $\left(\left(\mathcal{H}_{A} \otimes \mathcal{H}_{B}\right) \oplus\left(\mathcal{H}_{A} \otimes \mathcal{H}_{C}\right),\left(\pi_{A} \otimes\right.\right.$ $\left.\left.\pi_{B}\right) \oplus\left(\pi_{A} \otimes \pi_{C}\right)\right)$ is a faithful representation of $(A \otimes B) \oplus(A \otimes C)$. Then, to prove $\left\langle\mathrm{id} \odot \pi_{1}\right.$, id $\left.\odot \pi_{2}\right\rangle$ is an isometry, it suffices to show the following diagram commutes (because the other maps are isometries).

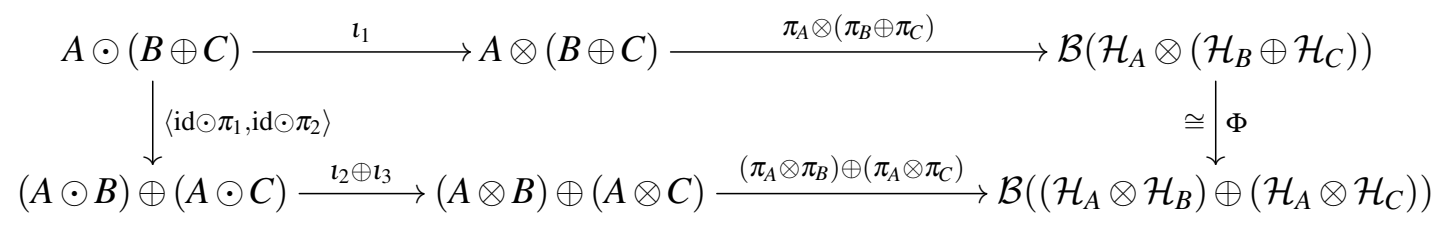

Here $\Phi$ is a $*$-isomorphism induced by an isomorphism $\phi: \mathcal{H}_{A} \otimes\left(\mathcal{H}_{B} \oplus \mathcal{H}_{C}\right) \rightarrow\left(\mathcal{H}_{A} \otimes \mathcal{H}_{B}\right) \oplus\left(\mathcal{H}_{A} \otimes \mathcal{H}_{C}\right)$ of Hilbert spaces. For $a \in A, b \in B, c \in C$,

$$
\begin{aligned}
& \left(\Phi \circ\left(\pi_{A} \otimes\left(\pi_{B} \oplus \pi_{C}\right)\right) \circ \boldsymbol{l}_{1}\right)(a \otimes(b, c)) \\
& =\Phi\left(\pi_{A}(a) \otimes\left(\pi_{B}(b) \oplus \pi_{C}(c)\right)\right) \\
& =\phi \circ\left(\pi_{A}(a) \otimes\left(\pi_{B}(b) \oplus \pi_{C}(c)\right)\right) \circ \phi^{-1} \\
& \stackrel{\star}{=}\left(\pi_{A}(a) \otimes \pi_{B}(b)\right) \oplus\left(\pi_{A}(a) \otimes \pi_{C}(c)\right) \\
& =\left(\left(\pi_{A} \otimes \pi_{B}\right) \oplus\left(\pi_{A} \otimes \pi_{C}\right)\right)(a \otimes b, a \otimes c) \\
& =\left(\left(\left(\pi_{A} \otimes \pi_{B}\right) \oplus\left(\pi_{A} \otimes \pi_{C}\right)\right) \circ\left(l_{2} \oplus l_{3}\right) \circ\left\langle\operatorname{id} \odot \pi_{1}, \mathrm{id} \odot \pi_{2}\right\rangle\right)(a \otimes(b, c)),
\end{aligned}
$$

where the marked equality $\stackrel{\star}{=}$ is showed by checking the commutativity of the following diagram.

$$
\begin{aligned}
& \mathcal{H}_{A} \otimes\left(\mathcal{H}_{B} \oplus \mathcal{H}_{C}\right) \stackrel{\phi}{\cong}\left(\mathcal{H}_{A} \otimes \mathcal{H}_{B}\right) \oplus\left(\mathcal{H}_{A} \otimes \mathcal{H}_{C}\right) \\
& \pi_{A}(a) \otimes\left(\pi_{B}(b) \oplus \pi_{C}(c)\right) \downarrow \mid\left(\pi_{A}(a) \otimes \pi_{B}(b)\right) \oplus\left(\pi_{A}(a) \otimes \pi_{C}(c)\right) \\
& \mathcal{H}_{A} \otimes\left(\mathcal{H}_{B} \oplus \mathcal{H}_{C}\right) \stackrel{\phi}{\cong}\left(\mathcal{H}_{A} \otimes \mathcal{H}_{B}\right) \oplus\left(\mathcal{H}_{A} \otimes \mathcal{H}_{C}\right)
\end{aligned}
$$


Theorem B.6. Let $M, N, L$ be $W^{*}$-algebras. Then the canonical maps

$$
\begin{gathered}
!: M \bar{\otimes} 0 \longrightarrow 0 \\
\left\langle\operatorname{id} \bar{\otimes} \pi_{1}, \mathrm{id} \bar{\otimes} \pi_{2}\right\rangle: M \bar{\otimes}(N \oplus L) \longrightarrow(M \bar{\otimes} N) \oplus(M \bar{\otimes} L)
\end{gathered}
$$

are $*$-isomorphisms.

Proof. Note that $0_{*} \cong 0$. Hence $M_{*} \otimes 0_{*} \cong M_{*} \odot 0_{*} \cong 0$ and $M \bar{\otimes} 0=\left(M_{*} \otimes 0_{*}\right)^{*} \cong 0$, which proves the first one.

We will show the latter one. Note that their preduals are explicitly given:

$$
\begin{aligned}
&(M \bar{\otimes}(N \oplus L))_{*} \cong M_{*} \otimes(N \oplus L)_{*} \cong M_{*} \otimes\left(N_{*} \oplus^{1} L_{*}\right) \\
&((M \bar{\otimes} N) \oplus(M \bar{\otimes} L))_{*} \cong(M \bar{\otimes} N)_{*} \oplus^{1}(M \bar{\otimes} L)_{*} \cong\left(M_{*} \otimes N_{*}\right) \oplus^{1}\left(M_{*} \otimes L_{*}\right)
\end{aligned}
$$

So, we will first show $M_{*} \otimes\left(N_{*} \oplus^{1} L_{*}\right) \cong\left(M_{*} \otimes N_{*}\right) \oplus^{1}\left(M_{*} \otimes L_{*}\right)$. For this, by a similar argument to the proof of Theorem B.5, it suffices to show $\left\langle\right.$ id $\odot \pi_{1}$, id $\left.\odot \pi_{2}\right\rangle: M_{*} \odot\left(N_{*} \oplus^{1} L_{*}\right) \rightarrow\left(M_{*} \odot N_{*}\right) \oplus^{1}\left(M_{*} \odot L_{*}\right)$ is an isometry. This follows from the commutativity of the following diagram, using Lemma B.3.

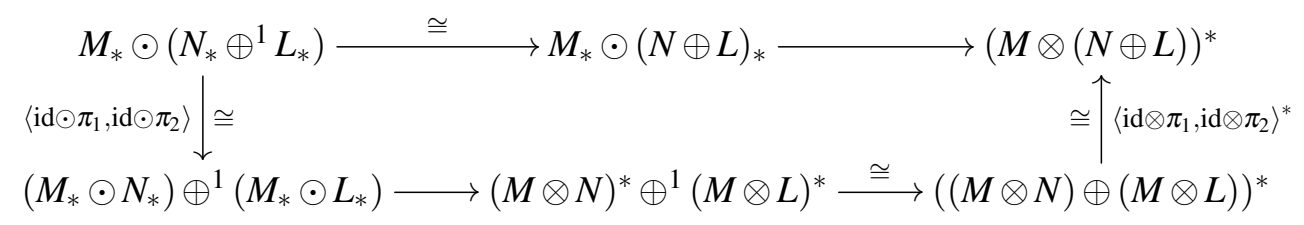

Hence we have an isometric isomorphism $\left\langle\mathrm{id} \otimes \pi_{1}, \mathrm{id} \otimes \pi_{2}\right\rangle: M_{*} \otimes\left(N_{*} \oplus^{1} L_{*}\right) \rightarrow\left(M_{*} \otimes N_{*}\right) \oplus^{1}\left(M_{*} \otimes L_{*}\right)$. Now, we can see the canonical map $\left\langle\operatorname{id} \bar{\otimes} \pi_{1}, \mathrm{id} \bar{\otimes} \pi_{2}\right\rangle: M \bar{\otimes}(N \oplus L) \rightarrow(M \bar{\otimes} N) \oplus(M \bar{\otimes} L)$, i.e. $\left\langle\left(\mathrm{id}_{*} \otimes\right.\right.$ $\left.\left.\pi_{1 *}\right)^{*},\left(\mathrm{id}_{*} \bar{\otimes} \pi_{2 *}\right)^{*}\right\rangle:\left(M_{*} \otimes(N \oplus L)_{*}\right)^{*} \rightarrow\left(M_{*} \otimes N_{*}\right)^{*} \oplus\left(M_{*} \otimes L_{*}\right)^{*}$, is an isomorphism because we can check the following diagram commutes.

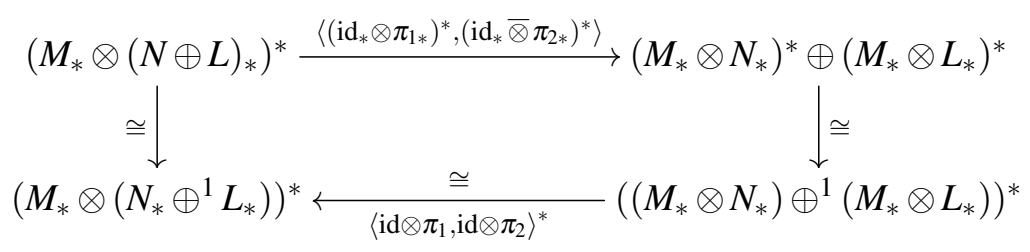

\section{Other omitted proofs}

\section{C.1 Miscellaneous results on $C^{*}$ - and $W^{*}$-algebras}

Here we show miscellaneous results on $C^{*}$ - and $W^{*}$-algebras, which is used in the main text or later in $\$$ C.2. Note again that, in this paper, $C^{*}$-algebras are always assumed to be unital.

For an element $x \in A$ of a $C^{*}$-algebra, we write $\operatorname{Sp}(x):=\{\lambda \in \mathbb{C} \mid x-\lambda 1$ is not invertible $\}$ for the spectrum of $x$. Here are several facts on spectrum in $C^{*}$-algebras.

Proposition C.1 ([43, Proposition I.4.2]). Let A be a $C^{*}$-algebra and $x \in A$ a normal element. Then the norm of $x$ coincides with the spectral radius of $x$, that is, $\|x\|=\sup _{\lambda \in \operatorname{Sp}(x)}|\lambda|$.

Proposition C.2 ([43, Proposition I.4.3 and Theorem I.6.1]). Let $A$ be a $C^{*}$-algebra and $x \in A$ a selfadjoint element. Then $\mathrm{Sp}(x) \subseteq \mathbb{R}$; and $\mathrm{Sp}(x) \subseteq \mathbb{R}^{+}$if and only if $x$ is positive. 
Proposition C.3. Let $A$ be a $C^{*}$-algebra and $x \in A$ a self-adjoint element. For any $M \in \mathbb{R}^{+}$, we have

$$
\|x\| \leq M \Longleftrightarrow-M 1 \leq x \leq M 1 .
$$

Proof. Note first that for any $\alpha, \beta \in \mathbb{C}(\alpha \neq 0)$ we have $\operatorname{Sp}(\alpha x+\beta 1)=\alpha \cdot \operatorname{Sp}(x)+\beta$. Then

$$
\begin{aligned}
\|x\| \leq M & \Longleftrightarrow \sup _{\lambda \in \operatorname{Sp}(x)}|\lambda| \leq M \\
& \Longleftrightarrow \forall \lambda \in \operatorname{Sp}(x) \cdot-M \leq \lambda \leq M \\
& \Longleftrightarrow \forall \lambda \in \operatorname{Sp}(x) \cdot M-\lambda \geq 0 \text { and } \lambda+M \geq 0 \\
& \Longleftrightarrow \operatorname{Sp}(M 1-x) \subseteq \mathbb{R}^{+} \text {and } \operatorname{Sp}(x+M 1) \subseteq \mathbb{R}^{+} \\
& \Longleftrightarrow M 1-x \geq 0 \text { and } x+M 1 \geq 0 \\
& \Longleftrightarrow-M 1 \leq x \leq M 1
\end{aligned}
$$

by Proposition C.2

As a special case we have $-\|x\| 1 \leq x \leq\|x\| 1$ for any self-adjoint element $x$. Now the next proposition is an easy consequence.

Proposition C.4. Let $A$ be a $C^{*}$-algebra and $S \subseteq A_{\mathrm{sa}}$ be a set of self-adjoint elements of $A$. Then $S$ is norm-bounded if and only if $S$ is order-theoretically bounded in $A_{\mathrm{sa}}$.

We will show some results on the spatial $W^{*}$-tensor products.

Lemma C.5. Let $A$ be a finite dimensional $W^{*}$-algebra (note that $A_{*}=A^{*}$ ), and let $M$ be a $W^{*}$-algebra. Then, the algebraic tensor product $A^{*} \odot M_{*}$ is already complete under the dual spatial $C^{*}$-norm. Moreover, the canonical embedding

$$
A \odot M \hookrightarrow\left(A^{*} \odot M_{*}\right)^{*}
$$

is surjective, so that $A \odot M \cong\left(A^{*} \odot M_{*}\right)^{*}$. Therefore, $A \odot M$ is a $W^{*}$-algebra with the predual $A^{*} \odot M_{*}$.

Proof. Fix a normalised basis $\left\{a_{1}, \ldots, a_{n}\right\}$ of $A$. We denote its dual basis by $\left\{\hat{a}_{1}, \ldots, \hat{a}_{n}\right\}$, which is a basis of $A^{*}$. Note that every element $\chi \in A^{*} \odot M_{*}$ is uniquely written as $\chi=\sum_{i=1}^{n} \hat{a}_{i} \otimes \varphi_{i}$. For arbitrary $x \in M$ with $\|x\| \leq 1$, we have $\left\|a_{i} \otimes x\right\|=\left\|a_{i}\right\|\|x\|=\|x\| \leq 1$ because any $C^{*}$-norm is a cross-norm. Hence

$$
\left|\varphi_{i}(x)\right|=\left|\left(\sum_{i=1}^{n} \hat{a}_{i} \otimes \varphi_{i}\right)\left(a_{i} \otimes x\right)\right|=\left|\chi\left(a_{i} \otimes x\right)\right| \leq \sup \{|\chi(z)| \mid z \in A \otimes M,\|z\| \leq 1\}=\|\chi\|,
$$

so that $\left\|\varphi_{i}\right\|=\sup \left\{\left|\varphi_{i}(x)\right| \mid x \in M,\|x\| \leq 1\right\} \leq\|\chi\|$ for each $i$. Now, assume that $\left(\chi_{j}\right)_{j}=\left(\sum_{i=1}^{n} \hat{a}_{i} \otimes \varphi_{i j}\right)$ is a Cauchy sequence in $A^{*} \odot M_{*}$. Because

$$
\left\|\varphi_{i k}-\varphi_{i j}\right\| \leq\left\|\sum_{i=1}^{n} \hat{a}_{i} \otimes\left(\varphi_{i k}-\varphi_{i j}\right)\right\|=\left\|\chi_{k}-\chi_{j}\right\|
$$

$\left(\varphi_{i j}\right)_{j}$ is a Cauchy sequence for each $i$. Let $\varphi_{i}=\lim _{j \rightarrow \infty} \varphi_{i j}$ and $\chi=\sum_{i=1}^{n} \hat{a}_{i} \otimes \varphi_{i}$. Then

$$
\left\|\chi-\chi_{j}\right\|=\left\|\sum_{i=1}^{n} \hat{a}_{i} \otimes\left(\varphi_{i}-\varphi_{i j}\right)\right\| \leq \sum_{i=1}^{n}\left\|\hat{a}_{i}\right\|\left\|\varphi_{i}-\varphi_{i j}\right\| \rightarrow 0 \quad \text { when } j \rightarrow \infty
$$

Hence $A^{*} \odot M_{*}$ is complete.

Let $\theta: A \odot M \rightarrow\left(A^{*} \odot M_{*}\right)^{*}$ be the canonical embedding. Take arbitrary $\Phi \in\left(A^{*} \odot M_{*}\right)^{*}$. For each $i$, define $\Phi_{i}: M_{*} \rightarrow \mathbb{C}$ by $\Phi_{i}(\varphi)=\Phi\left(\hat{a}_{i} \otimes \varphi\right)$. Clearly $\Phi_{i}$ is linear, and bounded because

$$
\left\|\Phi_{i}(\varphi)\right\|=\|\Phi(\hat{a} \otimes \varphi)\| \leq\|\Phi\|\|\hat{a} \otimes \varphi\|=\|\Phi\|\|\hat{a}\|\|\varphi\| .
$$


Hence $\Phi_{i} \in\left(M_{*}\right)^{*}$. Then we have $\theta\left(\sum_{i=1}^{n} a_{i} \otimes \imath^{-1}\left(\Phi_{i}\right)\right)=\Phi$, where $\imath: M \rightarrow\left(M_{*}\right)^{*}$ is the canonical isomorphism, because

$$
\theta\left(\sum_{i=1}^{n} a_{i} \otimes \imath^{-1}\left(\Phi_{i}\right)\right)\left(\hat{a}_{j} \otimes \varphi\right)=\sum_{i=1}^{n} \hat{a}_{j}\left(a_{i}\right) \varphi\left(\imath^{-1}\left(\Phi_{i}\right)\right)=\varphi\left(\imath^{-1}\left(\Phi_{j}\right)\right)=\Phi_{j}(\varphi)=\Phi\left(\hat{a}_{j} \otimes \varphi\right) .
$$

For a $*$-algebra $A$ and $n \in \mathbb{N}$, let $\mathcal{M}_{n}(A)$ denote the $*$-algebras of $n \times n$ matrices with entries from $A$. It is known that if $A$ is a $C^{*}$-algebra, then $\mathcal{M}_{n}(A)$ is a $C^{*}$-algebra. Because of the obvious $*$-isomorphism $\mathcal{M}_{n}(A) \cong \mathcal{M}_{n}(\mathbb{C}) \odot A$, the following is an immediate consequence of the above.

Corollary C.6. If $M$ is a $W^{*}$-algebra, then $\mathcal{M}_{n}(M)$ is a $W^{*}$-algebra.

Lemma C.7. In the setting of Lemma C.5 fix a basis $\left\{a_{1}, \ldots, a_{n}\right\}$ of A. Let $\left(z_{j}\right)_{j}=\left(\sum_{i=1}^{n} a_{i} \otimes x_{i j}\right)_{j}$ be a net in $A \odot M$, and let $z=\sum_{i=1}^{n} a_{i} \otimes x_{i} \in A \odot M$. Then, $z_{j} \rightarrow z$ ultraweakly in $A \odot M$ if and only if $x_{i j} \rightarrow x_{i}$ ultraweakly in $M$ for all $i \in\{1, \ldots, n\}$.

Proof. Assume that $z_{j} \rightarrow z$ ultraweakly in $A \odot M$. It means for all $\chi \in A^{*} \odot M_{*}$ one has $\chi\left(z_{j}\right) \rightarrow \chi(z)$. Then for all $i$ and for all $\varphi \in M_{*}$,

$$
\varphi\left(x_{i j}\right)=\left(\hat{a}_{i} \otimes \varphi\right)\left(\sum_{i=1}^{n} a_{i} \otimes x_{i j}\right) \rightarrow\left(\hat{a}_{i} \otimes \varphi\right)\left(\sum_{i=1}^{n} a_{i} \otimes x_{i}\right)=\varphi\left(x_{i}\right),
$$

that is, $x_{i j} \rightarrow x_{i}$ ultraweakly.

Conversely, assume $x_{i j} \rightarrow x_{i}$ ultraweakly in $M$ for all $i \in\{1, \ldots, n\}$. Then, for $\sum_{i=1}^{n} \hat{a}_{i} \otimes \varphi_{i} \in A^{*} \otimes M_{*}$,

$$
\begin{aligned}
\left|\left(\sum_{i=1}^{n} \hat{a}_{i} \otimes \varphi_{i}\right)\left(z-z_{j}\right)\right| & =\left|\left(\sum_{i=1}^{n} \hat{a}_{i} \otimes \varphi_{i}\right)\left(\sum_{i=1}^{n} a_{i} \otimes\left(x_{i}-x_{i j}\right)\right)\right| \\
& =\left|\sum_{i=1}^{n} \varphi_{i}\left(x_{i}-x_{i j}\right)\right| \\
& \leq \sum_{i=1}^{n}\left|\varphi_{i}\left(x_{i}\right)-\varphi_{i}\left(x_{i j}\right)\right| \rightarrow 0
\end{aligned}
$$

because $\varphi_{i}\left(x_{i j}\right) \rightarrow \varphi_{i}\left(x_{i}\right)$ for all $i$. Hence $z_{j} \rightarrow z$ ultraweakly in $A \odot M$.

Corollary C.8. Let $M$ be a $W^{*}$-algebra. Let $\left(x_{j}\right)_{j}=\left(\left[x_{k l j}\right]_{k l}\right)_{j}$ be a net in $\mathcal{M}_{n}(M)$, and let $x=\left[x_{k l}\right]_{k l} \in$ $\mathcal{M}_{n}(M)$. Then, $x_{j} \rightarrow x$ ultraweakly in $\mathcal{M}_{n}(M)$ if and only if $x_{k l j} \rightarrow x_{k l}$ ultraweakly in $M$ for all $k, l \in$ $\{1, \ldots, n\}$. In other words, one has $\mathrm{uw}^{-\lim _{j}}\left[x_{k l j}\right]_{k l}=\left[\mathrm{uw}-\lim _{j} x_{k l j}\right]_{k l}$, where uw-lim denotes the ultraweak limit.

Lemma C.9. Let $M, N$ be $W^{*}$-algebra. Let $x \in M$, and assume that a norm-bounded net $\left(y_{i}\right)_{i}$ converges ultraweakly to $y$ in $N$. Then a net $\left(x \otimes y_{i}\right)_{i}$ converges ultraweakly to $x \otimes y$ in $M \bar{\otimes} N$.

Proof. Recall that

$$
\begin{aligned}
y_{i} \rightarrow y \text { ultraweakly in } N \Longleftrightarrow \forall \varphi \in N_{*} \cdot \varphi\left(y_{i}\right) \rightarrow \varphi(y) \\
x \otimes y_{i} \rightarrow x \otimes y \text { ultraweakly in } M \bar{\otimes} N \Longleftrightarrow \forall \xi \in(M \bar{\otimes} N)_{*} \cdot \xi\left(y_{i}\right) \rightarrow \xi(y) .
\end{aligned}
$$

Then, for any $\varphi \in M_{*}$ and $\psi \in N_{*}$,

$$
\begin{aligned}
(\varphi \otimes \psi)\left(x \otimes y_{i}\right) & =\varphi(x) \cdot \psi\left(y_{i}\right) \\
& \rightarrow \varphi(x) \cdot \psi(y)=(\varphi \otimes \psi)(x \otimes y),
\end{aligned}
$$


because $\psi\left(y_{i}\right) \rightarrow \psi(y)$. Hence we have $\chi\left(x \otimes y_{i}\right) \rightarrow \chi(x \otimes y)$ for all $\chi \in M_{*} \odot N_{*}$. Now, take arbitrary $\xi \in M_{*} \otimes N_{*} \cong(M \bar{\otimes} N)_{*}$. Then, there exists a sequence $\left(\chi_{j}\right)_{j}$ in $M_{*} \odot N_{*}$ convergent to $\xi$ under the dual spatial $C^{*}$-norm (therefore, we have an inequation like $\left.|\xi(z)| \leq\|\xi\|\|z\|\right)$. Then

$$
\begin{aligned}
& \left|\xi(x \otimes y)-\xi\left(x \otimes y_{i}\right)\right| \\
& \leq\left|\xi(x \otimes y)-\chi_{j}(x \otimes y)\right|+\left|\chi_{j}(x \otimes y)-\chi_{j}\left(x \otimes y_{i}\right)\right|+\left|\chi_{j}\left(x \otimes y_{i}\right)-\xi\left(x \otimes y_{i}\right)\right| \\
& \leq\left\|\xi-\chi_{j}\right\|\|x \otimes y\|+\left|\chi_{j}(x \otimes y)-\chi_{j}\left(x \otimes y_{i}\right)\right|+\left\|\xi-\chi_{j} \mid\right\| x \otimes y_{i} \| \\
& =\left\|\xi-\chi_{j}\right\|\|x\|\|y\|+\left|\chi_{j}(x \otimes y)-\chi_{j}\left(x \otimes y_{i}\right)\right|+\left\|\xi-\chi_{j}\right\|\|x\|\left\|y_{i}\right\| \\
& =\left|\chi_{j}(x \otimes y)-\chi_{j}\left(x \otimes y_{i}\right)\right|+\left\|\xi-\chi_{j}\right\|\|x\|\left(\|y\|+\left\|y_{i}\right\|\right) .
\end{aligned}
$$

Take arbitrary $\varepsilon>0$. Because $\left(y_{i}\right)_{i}$ is norm-bounded, and $\chi_{j} \rightarrow \xi$, we have

$$
\left|\chi_{j}(x \otimes y)-\chi_{j}\left(x \otimes y_{i}\right)\right|+\left\|\xi-\chi_{j}\left|\|\| x \|\left(\|y\|+\left\|y_{i}\right\|\right)<\right| \chi_{j}(x \otimes y)-\chi_{j}\left(x \otimes y_{i}\right) \mid+\varepsilon\right.
$$

for large enough $j$. Finally, since $\chi_{j}\left(x \otimes y_{i}\right) \rightarrow \chi_{j}(x \otimes y)$, we have

$$
\left|\xi(x \otimes y)-\xi\left(x \otimes y_{i}\right)\right|<\left|\chi_{j}(x \otimes y)-\chi_{j}\left(x \otimes y_{i}\right)\right|+\varepsilon<2 \varepsilon
$$

for sufficiently large $i$. This proves $\xi\left(x \otimes y_{i}\right) \rightarrow \xi(x \otimes y)$. Hence $x \otimes y_{i}$ converges ultraweakly to $x \otimes y$ in $M \bar{\otimes} N$.

\section{C.2 Proofs for Dcppo -enrichment of Wstar $_{\mathrm{CPSU}}$}

Lemma C.10. The map $f$ defined in the proof of Proposition 5.2 satisfies $f(x)=\operatorname{uw}^{-\lim _{i}} f_{i}(x)$ for all $x \in M$.

Proof. By the definition of $f$ and by Proposition 4.4, we have $f(x):=\sup _{i} f_{i}(x)=\operatorname{uw}_{-} \lim _{i} f_{i}(x)$ for $x \in[0,1]_{M}$. Because of the linearity and the ultraweak continuity of the addition and the multiplication, we obtain $f(x)=\mathrm{uw}^{-\lim _{i}} f_{i}(x)$ for all $x \in M$.

Proposition C.11. The map $f$ defined in the proof of Proposition 5.2 is completely positive, and the supremum of $\left(f_{i}\right)_{i}$.

Proof. Consider $\mathcal{M}_{n}(f): \mathcal{M}_{n}(M) \rightarrow \mathcal{M}_{n}(N)$. For positive $\left[x_{k l}\right]_{k l} \in \mathcal{M}_{n}(M)$,

$$
\begin{aligned}
\mathcal{M}_{n}(f)\left(\left[x_{k l}\right]_{k l}\right) & =\left[f\left(x_{k l}\right)\right]_{k l} & & \\
& =\left[\mathrm{uw}-\lim _{i} f_{i}\left(x_{k l}\right)\right]_{k l} & & \text { by Lemma C.10 } \\
& =\mathrm{uw}_{-} \lim _{i}\left[f_{i}\left(x_{k l}\right)\right]_{k l} & & \text { by Corollary C.8 } \\
& =\mathrm{uw}_{-} \lim _{i} \mathcal{M}_{n}\left(f_{i}\right)\left(\left[x_{k l}\right]_{k l}\right) . & &
\end{aligned}
$$$$
=\mathrm{uw}_{-} \lim _{i}\left[f_{i}\left(x_{k l}\right)\right]_{k l} \quad \text { by Corollary C.8 }
$$

Now, $f_{i}$ is completely positive, so that $\mathcal{M}_{n}\left(f_{i}\right)\left(\left[x_{k l}\right]_{k l}\right)$ is positive for all $i$. Moreover, $f_{i} \sqsubseteq f_{j}$ implies $\mathcal{M}_{n}\left(f_{i}\right)\left(\left[x_{k l}\right]_{k l}\right) \leq \mathcal{M}_{n}\left(f_{j}\right)\left(\left[x_{k l}\right]_{k l}\right)$. Hence $\left(\mathcal{M}_{n}\left(f_{i}\right)\left(\left[x_{k l}\right]_{k l}\right)\right)_{i}$ is a positive monotone net in $\mathcal{M}_{n}(N)$, which is bounded because each $f_{i}$ is subunital and so is $M_{n}\left(f_{i}\right)$. By Proposition 4.4 (and Corollary C.6) we obtain

$$
\mathcal{M}_{n}(f)\left(\left[x_{k l}\right]_{k l}\right)=\mathrm{uw}_{-} \lim _{i} \mathcal{M}_{n}\left(f_{i}\right)\left(\left[x_{k l}\right]_{k l}\right)=\sup _{i} \mathcal{M}_{n}\left(f_{i}\right)\left(\left[x_{k l}\right]_{k l}\right) .
$$

Thus $\mathcal{M}_{n}(f)\left(\left[x_{k l}\right]_{k l}\right) \geq \mathcal{M}_{n}\left(f_{i}\right)\left(\left[x_{k l}\right]_{k l}\right) \geq 0$, so that $f$ is completely positive and $f_{i} \sqsubseteq f$ for all $i$. Let $f^{\prime} \in$ $\operatorname{Wstar}_{\mathrm{CPSU}}(M, N)$ with $f_{i} \sqsubseteq f^{\prime}$ for all $i$. Then, for positive $\left[x_{k l}\right]_{k l} \in \mathcal{M}_{n}(M)$, we have $\mathcal{M}_{n}\left(f_{i}\right)\left(\left[x_{k l}\right]_{k l}\right) \leq$ $\mathcal{M}_{n}\left(f^{\prime}\right)\left(\left[x_{k l}\right]_{k l}\right)$ for all $i$. Hence $\mathcal{M}_{n}(f)\left(\left[x_{k l}\right]_{k l}\right)=\sup _{i} \mathcal{M}_{n}\left(f_{i}\right)\left(\left[x_{k l}\right]_{k l}\right) \leq \mathcal{M}_{n}\left(f^{\prime}\right)\left(\left[x_{k l}\right]_{k l}\right)$. It follows that $f \sqsubseteq f^{\prime}$. 
Note that now Lemma C.10 gives a explicit formula $\left(\bigsqcup_{i} f_{i}\right)(x)=\mathrm{uw}-\lim _{i} f_{i}(x)$ for all $x \in M$.

Lemma C.12 ([1], Lemma 3.2.6]). Let $P, Q, R$ be posets. Then a map $f: P \times Q \rightarrow R$ is Scott-continuous if and only if it is separately Scott-continuous.

Proposition C.13. Let $M, N, L$ be $W^{*}$-algebras. The composition

$$
\circ: \operatorname{Wstar}_{\mathrm{CPSU}}(N, L) \times \mathbf{W s t a r}_{\mathrm{CPSU}}(M, N) \longrightarrow \mathbf{W s t a r}_{\mathrm{CPSU}}(M, L)
$$

is bi-strict Scott-continuous.

Proof. The bi-strictness is obvious because bottom maps $\perp$ are the zero maps. By Lemma C.12, it suffices to show the Scott-continuity in each variable. Let $\left(g_{i}\right)_{i}$ be a monotone net in $\operatorname{Wstar}_{\mathrm{CPSU}}(N, L)$ and let $f \in \mathbf{W} \operatorname{star}_{\mathrm{CPSU}}(M, N)$. It is easy to see $(-) \circ f$ is monotone, and hence $\left(g_{i} \circ f\right)_{i}$ is a monotone net in $\mathbf{W s t a r}_{\mathrm{CPSU}}(M, L)$. Then for each $x \in[0,1]_{M}$,

$$
\left(\left(\bigsqcup_{i} g_{i}\right) \circ f\right)(x)=\left(\bigsqcup_{i} g_{i}\right)(f(x))=\sup _{i} g_{i}(f(x))=\sup _{i}\left(g_{i} \circ f\right)(x)=\left(\bigsqcup_{i}\left(g_{i} \circ f\right)\right)(x)
$$

so that $\left(\bigsqcup_{i} g_{i}\right) \circ f=\bigsqcup_{i}\left(g_{i} \circ f\right)$.

Let $\left(f_{i}\right)_{i}$ be a monotone net in $\mathbf{W s t a r}_{\mathrm{CPSU}}(M, N)$ and let $g \in \mathbf{W} \operatorname{star}_{\mathrm{CPSU}}(N, L)$. It is easy to see $g \circ(-)$ is monotone, and hence $\left(g \circ f_{i}\right)_{i}$ is a monotone net in $\mathbf{W s t a r}_{\mathrm{CPSU}}(M, L)$. Then for each $x \in[0,1]_{M}$,

$$
\left(g \circ\left(\bigsqcup_{i} f_{i}\right)\right)(x)=g\left(\sup _{i} f_{i}(x)\right)=\sup _{i} g\left(f_{i}(x)\right)=\sup _{i}\left(g \circ f_{i}\right)(x)=\left(\bigsqcup_{i}\left(g \circ f_{i}\right)\right)(x),
$$

where we used the normality of $g$ (and Proposition 4.5) for the second equality. It shows $g \circ\left(\bigsqcup_{i} f_{i}\right)=$ $\bigsqcup_{i}\left(g \circ f_{i}\right)$.

Proposition C.14. Let $M, N, L$ be $W^{*}$-algebras. Then the tupling of maps

$$
\langle\cdot, \cdot\rangle: \mathbf{W s t a r}_{\mathrm{CPSU}}(L, M) \times \mathbf{W s t a r}_{\mathrm{CPSU}}(L, N) \stackrel{\cong}{\longrightarrow} \operatorname{Wstar}_{\mathrm{CPSU}}(L, M \oplus N)
$$

is strict Scott-continuous.

Proof. The strictness is easy. Because of the symmetry, and by Lemma C.12, it suffices to show the Scott-continuity in the first variable. Let $\left(f_{i}\right)_{i}$ be a monotone net in $\operatorname{Wstar}_{\mathrm{CPSU}}(L, M)$ and let $g \in \mathbf{W s t a r}_{\mathrm{CPSU}}(L, N)$. It is easy to see $\langle\cdot, g\rangle$ is monotone, and hence $\left(\left\langle f_{i}, g\right\rangle\right)_{i}$ is a monotone net in $\operatorname{Wstar}_{\mathrm{CPSU}}(L, M \oplus N)$. Then for each $x \in[0,1]_{L}$,

$$
\begin{aligned}
\left(\bigsqcup_{i}\left\langle f_{i}, g\right\rangle\right)(x) & =\sup _{i}\left\langle f_{i}, g\right\rangle(x) \\
& =\sup _{i}\left(f_{i}(x), g(x)\right) \\
& \left.=\sup _{i} f_{i}(x), \sup _{i} g(x)\right) \\
& =\left(\left(\bigsqcup_{i} f_{i}\right)(x), g(x)\right) \\
& =\left\langle\bigsqcup_{i} f_{i}, g\right\rangle(x) .
\end{aligned}
$$

Note that the order in $M \oplus N$ is pointwise, therefore the supremum is pointwise, too. Hence $\bigsqcup_{i}\left\langle f_{i}, g\right\rangle=$ $\left\langle\bigsqcup_{i} f_{i}, g\right\rangle$.

Proposition C.15. Let $M, M^{\prime}, N, N^{\prime}$ be $W^{*}$-algebras. The tensor product of maps

$$
\bar{\otimes}: \operatorname{Wutar}_{\mathrm{CPSU}}\left(M, M^{\prime}\right) \times \mathbf{W s t a r}_{\mathrm{CPSU}}\left(N, N^{\prime}\right) \longrightarrow \operatorname{Wstar}_{\mathrm{CPSU}}\left(M \bar{\otimes} N, M^{\prime} \bar{\otimes} N^{\prime}\right)
$$

is bi-strict Scott-continuous. 
Proof. Let $f \in \mathbf{W s t a r}_{\mathrm{CPSU}}\left(M, M^{\prime}\right)$. By the symmetry and Lemma C.12, it suffices to show

$$
f \bar{\otimes}(-): \mathbf{W s t a r}_{\mathrm{CPSU}}\left(N, N^{\prime}\right) \rightarrow \mathbf{W s t a r}_{\mathrm{CPSU}}\left(M \bar{\otimes} N, M^{\prime} \bar{\otimes} N^{\prime}\right)
$$

is strict Scott-continuous. Let $\perp \in \mathbf{W s t a r}_{\mathrm{CPSU}}\left(N, N^{\prime}\right)$ be the least element, i.e. the zero map. Then

$$
(f \bar{\otimes} \perp)(x \otimes y)=f(x) \otimes \perp(y)=f(x) \otimes 0=0
$$

for all $x \in M, y \in N$. Hence $(f \bar{\otimes} \perp)(z)=0$ for all $z \in M \odot N$. Because $M \odot N$ is ultraweakly dense in $M \bar{\otimes} N$ and $f \bar{\otimes} \perp$ is normal (i.e. ultraweakly continuous), we obtain $(f \bar{\otimes} \perp)(z)=0$ for all $z \in M \bar{\otimes} N$. Therefore $f \bar{\otimes} \perp=\perp$.

Let $g, g^{\prime} \in \mathbf{W} \operatorname{star}_{\mathrm{CPSU}}\left(N, N^{\prime}\right)$ with $g \sqsubseteq g^{\prime}$. By definition $g^{\prime}-g$ is completely positive, and so is $f \bar{\otimes}\left(g^{\prime}-g\right)$. Notice that $f \bar{\otimes}\left(g^{\prime}-g\right)=f \bar{\otimes} g^{\prime}-f \bar{\otimes} g$ because they coincide on $M \odot N$. Then $f \bar{\otimes} g^{\prime}-f \bar{\otimes} g$ is completely positive, and $f \bar{\otimes} g \sqsubseteq f \bar{\otimes} g^{\prime}$. Hence $f \bar{\otimes}(-)$ is monotone.

Let $\left(g_{i}\right)_{i}$ be a monotone net in $\mathbf{W s t a r}_{\mathrm{CPSU}}\left(N, N^{\prime}\right)$. By the monotonicity $\left(f \bar{\otimes} g_{i}\right)_{i}$ is a monotone net in $\operatorname{Wstar}_{\mathrm{CPSU}}\left(M \bar{\otimes} N, M^{\prime} \bar{\otimes} N^{\prime}\right)$. By a similar argument above, to prove $f \bar{\otimes}\left(\bigsqcup_{i} g_{i}\right)=\bigsqcup_{i}\left(f \bar{\otimes} g_{i}\right)$, it suffice to show $\left(f \bar{\otimes}\left(\bigsqcup_{i} g_{i}\right)\right)(x \otimes y)=\left(\bigsqcup_{i} f \bar{\otimes} g_{i}\right)(x \otimes y)$ for all $x \in M, y \in N$. This is showed as follows.

$$
\begin{aligned}
\left(f \bar{\otimes}\left(\bigsqcup_{i} g_{i}\right)\right)(x \otimes y) & =f(x) \bar{\otimes}\left(\bigsqcup_{i} g_{i}\right)(y) \\
& =f(x) \otimes\left(\mathrm{uw}_{-}-\lim _{i} g_{i}(y)\right) \\
& =\mathrm{uw}-\lim _{i}\left(f(x) \otimes g_{i}(y)\right) \\
& =\mathrm{uw}_{-} \lim _{i}\left(f \bar{\otimes} g_{i}\right)(x \otimes y) \\
& =\left(\bigsqcup_{i} f \bar{\otimes} g_{i}\right)(x \otimes y)
\end{aligned}
$$

by Lemma C.10

by Lemma C.9

by Lemma C.10 